\title{
Childhood Obesity Evidence Base Project: A Systematic Review and Meta-Analysis of a New Taxonomy of Intervention Components to Improve Weight Status in Children 2-5 Years of Age, 2005-2019
}

Lori A.J. Scott-Sheldon, PhD, ${ }^{1,2}$ Larry V. Hedges, PhD, ${ }^{3}$ Chris Cyr, BS, ${ }^{4}$ Deborah Young-Hyman, PhD, Laura Kettel Khan, PhD, ${ }^{6}$ Mackenzie Magnus, MPH, MBA, ${ }^{4}$ Heather King, PhD, ${ }^{4}$ Sonia Arteaga, PhD, John Cawley, PhD, 8,9 Christina D. Economos, PhD, ${ }^{10}$ Debra Haire-Joshu, PhD, RN, ' Christine M. Hunter, PhD, ${ }^{5}$ Bruce Y. Lee, MD, MBA, ${ }^{12}$ Shiriki K. Kumanyika, PhD, MPH, ${ }^{13}$ Lorrene D. Ritchie, PhD, RD, ${ }^{14}$ Thomas N. Robinson, MD, MPH, ${ }^{15}$ and Marlene B. Schwartz, $\mathrm{PhD}^{16}$

\section{Abstract}

Objective: To evaluate the efficacy of childhood obesity interventions and conduct a taxonomy of intervention components that are most effective in changing obesity-related health outcomes in children $2-5$ years of age.

Methods: Comprehensive searches located 51 studies from 18,335 unique records. Eligible studies: (1) assessed children aged 2-5, living in the United States; (2) evaluated an intervention to improve weight status; (3) identified a same-aged comparison group; (4) measured BMI; and (5) were available between January 2005 and August 2019. Coders extracted study, sample, and intervention characteristics. Effect sizes [ESs; and 95\% confidence intervals (CIs)] were calculated by using random-effects models. Metaregression was used to determine which intervention components explain variability in ESs.

Results: Included were 51 studies evaluating 58 interventions $(N=29,085$; mean age $=4$ years; $50 \%$ girls $)$. Relative to controls, children receiving an intervention had a lower BMI at the end of the intervention $(g=0.10,95 \% \mathrm{CI}=0.02-0.18 ; k=55)$ and at the last follow-up $(g=0.17,95 \% \mathrm{CI}=0.04-0.30 ; k=14$; range $=18-143$ weeks $)$. Three intervention components moderated efficacy: engage caregivers in praise/encouragement for positive health-related behavior; provide education about the importance of screen time reduction to caregivers; and engage pediatricians/health care providers.

\footnotetext{
'Centers for Behavioral and Preventive Medicine, The Miriam Hospital, Providence, RI, USA.

${ }^{2}$ Department of Psychiatry and Human Behavior, Alpert School of Medicine, Brown University, Providence, RI, USA.

${ }^{3}$ Department of Statistics, Northwestern University, Evanston, IL, USA.

${ }^{4}$ Impact Genome Project, Mission Measurement, Chicago, IL, USA.

${ }^{5}$ Office of Behavioral and Social Sciences, Office of the Director, National Institutes of Health, Bethesda, MD, USA.

${ }^{6}$ Division of Nutrition, Physical Activity, and Obesity, Centers for Disease Control and Prevention, Atlanta, GA, USA.

${ }^{7}$ Office of the Director, National Institutes of Health, National Institutes of Health, Bethesda, MD, USA.

Departments of ${ }^{8}$ Policy Analysis and Management and ${ }^{9}$ Economics, Cornell University, Ithaca, NY, USA.

${ }^{10}$ Division of Nutrition Interventions, Communication, and Behavior Change, Friedman School of Nutrition Science and Policy, Tufts University, Boston, MA, USA.

${ }^{\prime \prime}$ Center for Obesity Prevention and Policy Research, Brown School, Washington University, Saint Louis, MO, USA.

${ }^{12}$ CUNY Graduate School of Public Health and Policy, New York, NY, USA.

${ }^{13}$ Department of Community Health and Prevention, Dornsife School of Public Health, Drexel University, Philadelphia, PA, USA.

${ }^{14}$ Nutrition Policy Institute, University of California, Division of Agriculture and Natural Resources, Berkeley, CA, USA.

${ }^{15}$ Departments of Pediatrics and Medicine, Stanford Solutions Science Lab, Stanford University, Stanford, CA, USA.

${ }^{16}$ Department of Human Development and Family Studies, University of Connecticut, Hartford, CT, USA.
} 
Conclusions: Early childhood obesity interventions are effective in reducing BMI in preschool children. Our findings suggest that facilitating caregiver education about the importance of screen time reduction may be an important strategy in reducing early childhood obesity.

Keywords: BMI; intervention; meta-analysis; prevention; taxonomy

\section{Introduction}

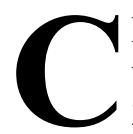
hildhood obesity is a major public health challenge, with one in three US children between the ages of 2 and 5 meeting criteria for overweight or obesity. ${ }^{1}$ The urgency to reverse the course of childhood obesity has led to significant growth in the scientific literature evaluating childhood obesity interventions. Extant reviews of this research have provided limited guidance regarding strategies to reduce the rates of childhood obesity among preschool-age children living in the United States, because most reviews include children from a wide age range (e.g., 2-18 years of age) and multiple countries; report varying outcomes measures (e.g., BMI, nutrition, and/or physical activity); or are limited to a specific intervention setting (e.g., schools). ${ }^{2-12}$ Traditional meta-analytic reviews often narrowly focus on the efficacy of childhood obesity interventions as a whole and do not address the conditions under which the intervention is most impactful.

The objective of this systematic review and meta-analysis is twofold: (1) to evaluate the scientific evidence regarding the efficacy of obesity interventions measuring BMI for children 2-5 years of age living in the United States and (2) to develop and apply a taxonomy of intervention components to identify specific components that increase the efficacy of these interventions in their context. Efficacy was measured as change in BMI, because it is the most commonly used indicator to assess overweight and obesity in children. ${ }^{13} \mathrm{We}$ hypothesized that children receiving interventions to prevent obesity or improve weight status would experience less gain in BMI, or perhaps reductions in BMI, relative to children comparable in age, who did not receive the intervention.

Understanding childhood obesity programs requires developing methods not only to address the question of whether a given intervention works but also to better understand what works, for whom, and under what conditions intervention efficacy is improved. ${ }^{14}$ Childhood obesity prevention interventions are often evaluated as a whole, as opposed to the specific elements that comprise the interventions, which hinders effective implementation and dissemination of evidence-based approaches to reduce obesity (cf Ma et al. ${ }^{15}$ ). Therefore, a taxonomy of intervention components was developed by using the grounded theory ${ }^{16,17}$ to identify the specific content of the interventions and considered within the socioecological model (SEM). ${ }^{18,19}$ (A detailed description of the taxonomy development is described in the Methods paper for this project. ${ }^{19}$ )

We also examined the extent to which the efficacy of the interventions depended on characteristics of the samples and methods. Hypothesized moderators included: (1) child demographic characteristics (e.g., proportion obese or overweight at baseline, race/ethnicity, and socioeconomic status); (2) elements of study or intervention design (e.g., recruitment site, implementation setting, intervention recipient, content focus, and duration); and (3) intervention components (e.g., provide education about nutrition and healthy eating patterns to caregivers, engage caregivers in supporting physical activities). We expected that childhood obesity interventions would be more effective when: (1) fewer children of low socioeconomic status or race/ ethnic minorities (as healthy lifestyle changes are challenging for caregivers and children experiencing financial hardship ${ }^{20}$ or living in disadvantaged neighborhoods ${ }^{21}$ ) or more children meeting criteria for overweight or obesity (given emerging evidence that individual differences in behavioral phenotypes for childhood obesity may impact the efficacy of obesity prevention and treatment interventions $^{22}$ ) were included in the study samples; (2) the sample was recruited from and the intervention was implemented in a school-based setting (as school-based interventions have been shown to be more effective in reducing BMI than interventions implemented in other settings, such as home) $;^{11}$ and (3) targeted both healthy nutrition and physical activity ${ }^{4}$ and involved a caregiver ${ }^{23}$ (caregiver refers to a parent, grandparent, other adult family members, or foster-parent who is directly responsible for the care of a child). Because the goal of a taxonomy is to identify intervention components as they emerge in the childhood obesity intervention programs, no hypotheses were generated about the efficacy of the specific individual or combination of components.

\section{Methods}

This systematic review and meta-analysis was performed by using established meta-analytic methods ${ }^{24,25}$ and reported in accordance with the Preferred Reporting Items for Systematic Reviews and Meta-Analyses (PRISMA) Statement. ${ }^{26}$ The PRISMA checklist (Supplementary Table S1) is available at https://www.nccor.org/childhoodobesity-evidence-base-test-of-a-novel-taxonomic-metaanalytic-method.

\section{Eligibility Criteria}

The study inclusion criteria were developed based on the Population, Interventions, Comparisons, Outcomes, and Study Design (PICOS) framework. ${ }^{27}$ Eligible studies: (1) assessed children aged 2-5 years at the beginning of the invention, living in the United States; (2) evaluated an intervention to prevent obesity in children; (3) identified a 
same-aged control or comparison group; (4) assessed BMI [weight $(\mathrm{kg}) /$ height $\left(\mathrm{m}^{2}\right)$ ], BMI percentile, or BMI z-score; and (5) were available (published or unpublished) between January 1, 2005 and August 31, 2019.

\section{Information Sources}

A comprehensive strategy was used to identify, locate, and retrieve available scientific research reports. (This search was independent of the search conducted in the development of the intervention component taxonomy. ${ }^{19}$ ) Our primary strategy involved searches of electronic bibliographic databases (e.g., PubMed, Embase). To supplement this primary strategy, we also reviewed relevant published reviews and meta-analyses, reference lists of manuscripts retrieved from our database searches, electronic databases of funded research (NIH RePORTER), and registered clinical trials (www.ClinicalTrials.gov); we consulted with members of the National Collaborative on Childhood Obesity Research (NCCOR) Childhood Obesity Evidence Base (COEB) Project Workgroup (WG) and External Expert Panel (EEP).

\section{Search Strategy}

The search string was developed based on the intended recipients and outcome of interest, and it included Boolean operators, wildcard symbols, and quotations that used a combination of the following broad terms: childhood, obesity, and intervention. Because some electronic databases have a controlled vocabulary thesaurus to index records [e.g., Medical Subject Heading (MeSH) terms used in PubMed], specific search parameters were developed for each database. The full search string used for each electronic database search can be found in Supplementary Data S1. A broad search of the childhood obesity literature was conducted rather than restricting the search to children 2-5 years of age to ensure retrieval of all studies that included the targeted age range (i.e., mean age 2 to $\leq 5$ years). No language, geographical region, or publication date restrictions were applied. All electronic bibliographic searches were conducted in June 2019. Records retrieved from each database were imported into a reference management software library (EndNote $\mathrm{X} 8^{28}$ ), and duplicate records were automatically removed.

\section{Study Selection}

All records retrieved from the electronic database searches were screened for inclusion based on the title and abstract by two reviewers. This initial screening was conducted to identify any additional duplicate records, irrelevant records, review papers, editorials, and commentaries. Full-text documents of potentially relevant records and references from manuscripts were reviewed based on the inclusion criteria. Registries of funded research and clinical trials were cross-checked with the relevant full-text manuscripts to ensure the retrieval of all available reports. If the study methods or data were reported in multiple documents, they were linked in the database and represented as a single study to avoid multiple/duplicate publication bias.
The manuscript reporting the most complete data was selected as the primary study; additional documents were considered supplemental (e.g., clinical trial record, protocol) but were consulted in the data collection process.

\section{Data Collection Process}

Two trained coders independently extracted relevant study information (e.g., publication year) from the primary study and supplemental manuscripts, as well as intended recipient characteristics (e.g., age, gender, race/ethnicity), design and measurement (e.g., random assignment), and intervention details (e.g., dose, number of sessions, length, and duration). In addition to the intended recipient and intervention context characteristic taxonomies developed for the COEB project, a taxonomy of intervention components with 93 distinct components, grouped into 9 categories, were used to identify the specific activity components of the intervention. ${ }^{19}$ For each intervention, the distinct components were coded as present (1) or absent (0). Intervention components were coded as absent if no clear evidence of the component could be identified from the intervention details provided in the primary study manuscript or the supplemental documents.

Disagreements between coders were reviewed, discussed, and reconciled by the coders; any unresolved disagreements were reviewed and finalized by the lead methodologist (L.A.J.S.S.). Inter-rater reliability was assessed across the study, sample, design, and intervention characteristics coded. For the categorical variables, coders agreed on $90 \%$ of the judgments (mean Cohen's $\kappa=0.68$; range $=0.20-1.00)$. For the continuous variables, the average intra-class correlation coefficient $(\rho)$ was 0.88 (median $=1.00)$.

\section{Risk of Bias in Individual Studies}

The Cochrane Risk of Bias Assessment Tool (Version 5.1.0) was used to assess the risk of bias in each study. ${ }^{29}$ The tool assesses seven sources of bias: random sequence generation, allocation concealment, blinding of participants and personnel, blinding of outcome assessment, incomplete outcome data, selective reporting, and other. The sources of bias are assigned a judgment of high, low, or unclear risk of bias when study details are insufficient to make a judgment of the level of risk.

\section{Study Outcomes}

The primary study outcome considered for this metaanalysis was change in BMI expressed as weight $(\mathrm{kg}) /$ height $\left(\mathrm{m}^{2}\right)$, percentile, or $\mathrm{z}$-score because it is the most commonly used indicator to assess overweight and obesity in children. ${ }^{13}$ Other discrete measures of BMI (e.g., overweight, obesity) were also included. Secondary outcomes such as measures of physical activity, diet, and sleep were identified during the taxonomy development and included in the outcomes taxonomy, but they were not included in the current meta-analysis due to inconsistent reporting methods and the limited number of studies including these measures. 


\section{Summary Measures}

Effect sizes (ESs) were calculated as the pretest-posttest change in BMI divided by the pretest standard deviation (SD) in BMI within each condition and then subtracting the control group from the intervention group. ${ }^{30,31}$ Thus, all ESs controlled for baseline. If a study reported proportions, an odds ratio was calculated and transformed to a standardized mean difference ES by using the Cox transformation. ${ }^{32}$ The sampling variance for each ES was calculated by using standard procedures, correcting for sample size bias (Hedges' $g$ ). ${ }^{31,33}$ Multiple ESs were calculated from individual studies when the study reported more than one outcome, timepoint, or intervention condition, or when outcomes were separated by sample characteristics (e.g., girls and boys). To avoid violating the assumption of independence, ${ }^{25,34}$ we: (1) assessed each outcome separately; (2) clustered the ESs by timepoint (end of intervention and last follow-up assessment); and (3) conducted sensitivity analyses to determine whether multiple comparisons from a single study affected the overall outcomes. ${ }^{34}$ An overall measure of BMI change was created by: (1) using the BMI metric reported among the studies reporting only one metric; and (2) averaging ESs from studies reporting more than one BMI metric (e.g., percentile, z-score). Positive ESs indicated smaller BMI increases over time among children who received the intervention relative to the comparison group.

\section{Synthesis of Results}

The distribution of the ESs for each dependent variable was inspected for possible outliers; extreme ESs (i.e., ESs more than 1.5 times the interquartile range beyond the 25 th and 75th percentiles) were removed, and the magnitude and direction of the pooled ESs with or without the outliers were compared. Hedges' $g$ (and corresponding 95\% CIs) were calculated by using random-effects procedures. The between-study variance was estimated by using full information maximum likelihood. ${ }^{35}$ The $95 \%$ CIs surrounding the weighted mean ES indicates whether the ES is statistically significant as well as the degree of precision. Heterogeneity in ESs was identified and quantified by computing $Q$ and the $I^{2}$ index. The $Q$ statistic has a chisquare distribution with degrees of freedom equal to $k-1$; a significant $Q$ indicates a lack of homogeneity and an inference of heterogeneity. To assess the extent to which outcomes were consistent across studies, the $I^{2}$ index and its corresponding $95 \%$ CIs were calculated. ${ }^{36,37} I^{2}$ values of $25 \%, 50 \%$, and $75 \%$ can be considered low, medium, and high heterogeneity, respectively. ${ }^{38}$ Data analyses were conducted in Stata ${ }^{39}$ by using published macros. ${ }^{25,40}$

\section{Risk of Bias across Studies}

Asymmetries in the distributions of ESs, indicating a possible publication bias, ${ }^{41}$ were examined by (1) inspecting funnel plots ${ }^{42}$ and (2) using regression ${ }^{43}$ and rank $^{44}$ tests assessing the degree of funnel plot asymmetry in the distribution of ESs. The trim-and-fill method ${ }^{45}$ was used to estimate and correct for the possibility of missing studies (using a nonparametric approach based on funnel plot asymmetry) if publication bias was detected. ${ }^{43,44}$

\section{Moderator Analyses}

Meta-regression was conducted to assess the association between study-level moderators and ESs. For these random-effect regression models, the inverse variance for each ES included error associated with within-study level sampling error and additional between-study population variance. ${ }^{46}$ Sample (proportion racial/ethnic minorities, socioeconomic status, proportion obese or overweight), study (recruitment site, implementation setting, intervention recipient, content focus, duration), and intervention (components as identified by our newly developed taxonomy $^{19}$ ) features were examined. Significant moderators were simultaneously entered in a multiple regression model, and they were adjusted for multiple testing by using random permutations. ${ }^{46}$

\section{Results}

\section{Study Selection}

Comprehensive searches of electronic bibliographic databases located 51 unique studies from 18,177 records with relevant key terms (after removing duplicates). An additional 158 records were identified through other sources (i.e., reviews, reference lists, funded research, clinical trial records, consultation with experts). Of the 18,335 unique records reviewed, 16,311 records were excluded based on title and abstract review because those records did not meet inclusion criteria or were reviews, editorials, or commentaries. An additional 1826 records were excluded after full-text review, because the studies did not meet inclusion criteria (Fig. 1). Importantly, a primary reason for study exclusion was failure to measure (or report) the primary outcome for this meta-analysis, BMI. The final sample included 51 unique studies and 147 supplemental documents that provided additional study details or data for the included studies. Nine supplemental documents provided additional information for more than one primary study (e.g., Hip Hop to Health manual ${ }^{47}$ ). Table 1 provides a description of the 51 included studies reporting on 58 interventions ( 7 studies evaluated 2 interventions ${ }^{48-54}$ ). Additional intervention details can be found in Supplementary Table S2.

\section{Study and Sample Characteristics}

Studies were published (or available) between 2005 and 2019 (mean publication date $=2015, \mathrm{SD}=4$ ), and they were typically conducted in the U.S. Midwest (29\%), Southeast (24\%), or Northeast (18\%). Most of the included studies $(88 \%)$ used a randomized controlled trial design with random assignment of groups $(49 \%)$ or individuals (39\%); six studies used a quasi-experimental design. ${ }^{52,54-58}$ Study samples were recruited from child care centers or preschools $(55 \%)$, clinical practices $(22 \%)$, community 


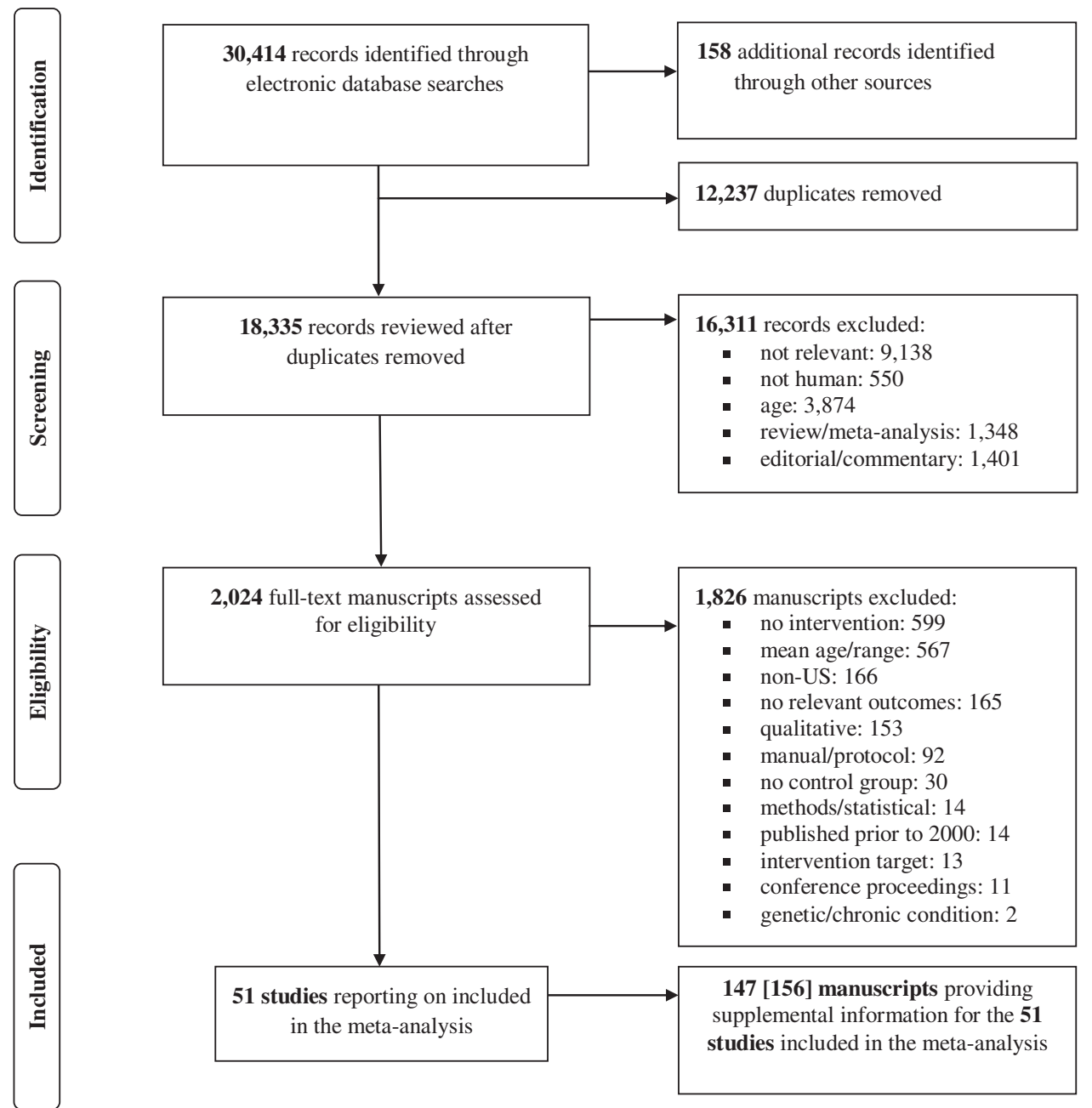

Figure 1. Childhood Obesity Evidence Base Systematic Review and Meta-Analysis Study Search and Selection Process.

sites/programs (14\%), or multiple school, clinic, or community sites (10\%). Participants included 29,085 children (mean age $=48$ months, $\mathrm{SD}=7$ ), with an average retention rate of $80 \%(\mathrm{SD}=0.18)$ across the studies. The study samples included, on average, $50 \%(\mathrm{SD}=0.27$; range $=20 \%-100 \%)$ of children meeting criteria for overweight or obesity. Details of the study and sample characteristics are provided in Table 2.

\section{Characteristics of Childhood Obesity Interventions}

Interventions were typically theory driven (79\%) and targeted both nutrition and physical activity $(48 \%)$, nutrition only $(14 \%)$, physical activity only $(5 \%)$, or multiple/other obesogenic behaviors $(33 \%$; e.g., screen time, sleep, behavioral self-management). The content of the intervention was most often delivered to the child and caregiver $(72 \%)$, caregiver alone $(17 \%)$, or the child $(10 \%)$. Dose varied by intervention target; that is, interventions were delivered to the child across a median of 21 sessions of $<1$ hour each (median $=53$ minutes), to the parent across a median of nine 1-hour sessions (median $=60$ minutes), and to the facilitator (e.g., teacher, physician) during a single 6-hour session (median $=360$ minutes). The average duration of the intensive intervention phase was 9 months $(\mathrm{SD}=7 ;$ range $=2-36$ months). Active control or comparison conditions (43\%) were used most often followed by no intervention (31\%), waitlist $(16 \%)$, or education only $(10 \%)$.

\section{Childhood Obesity Intervention Components Identified Using the Taxonomy}

A total of 90 (out of 93) intervention components were identified across the interventions included in the current meta-analysis. Three intervention components included in the taxonomy were not identified: (1) provided materials to support self-control in children; (2) implemented Earned Income Tax Credit (i.e., adoption or expansion of Earned Income Tax Credits); and (3) implemented policies regarding food/beverage costs. (These intervention components, generated in the taxonomy development phase, were not identified in our meta-analysis because we limited the inclusion criteria to studies measuring BMI where a standardized mean difference between the intervention and control group could be calculated.) 


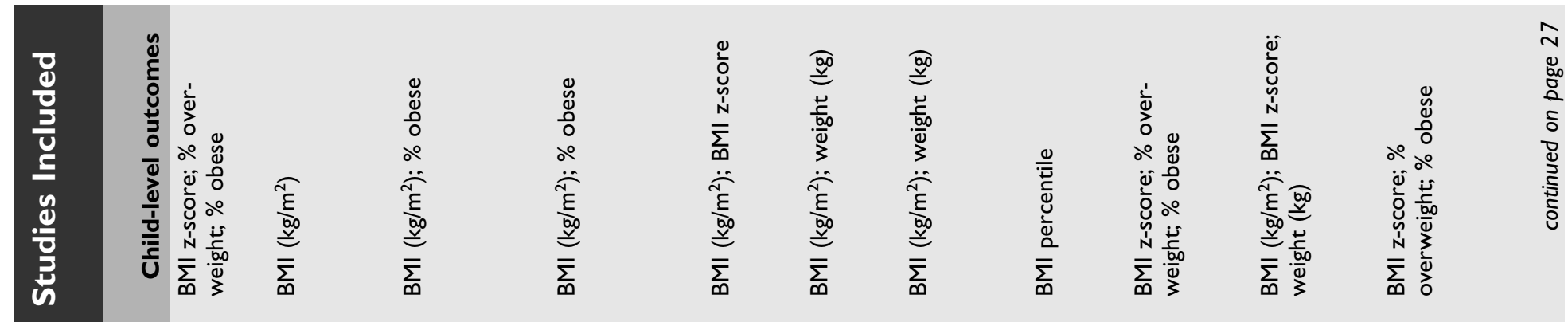

.

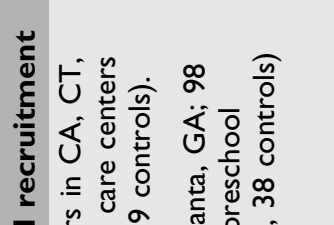

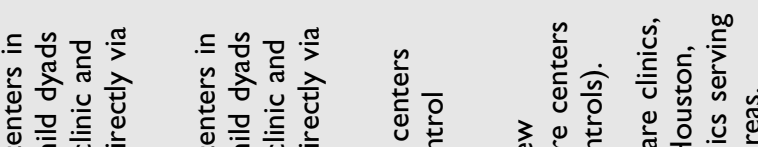

苍苋

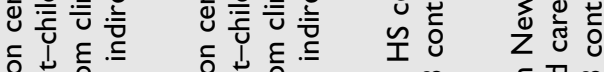

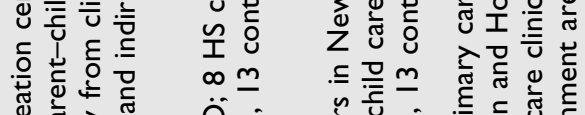

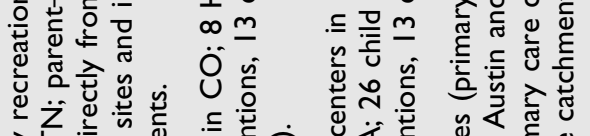

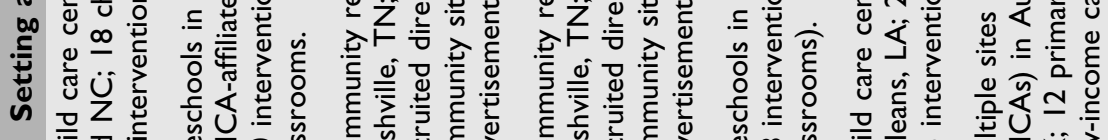

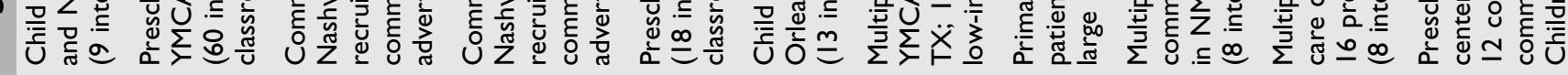

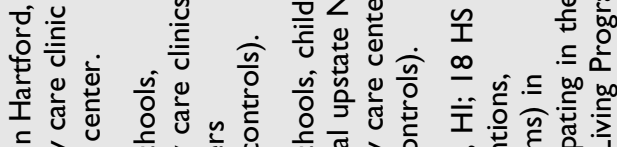

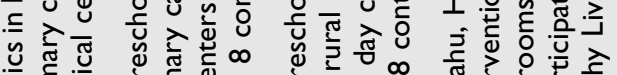

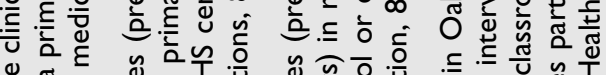

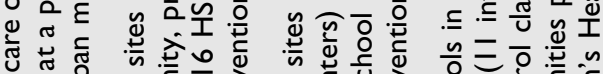

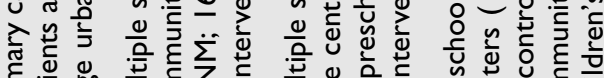

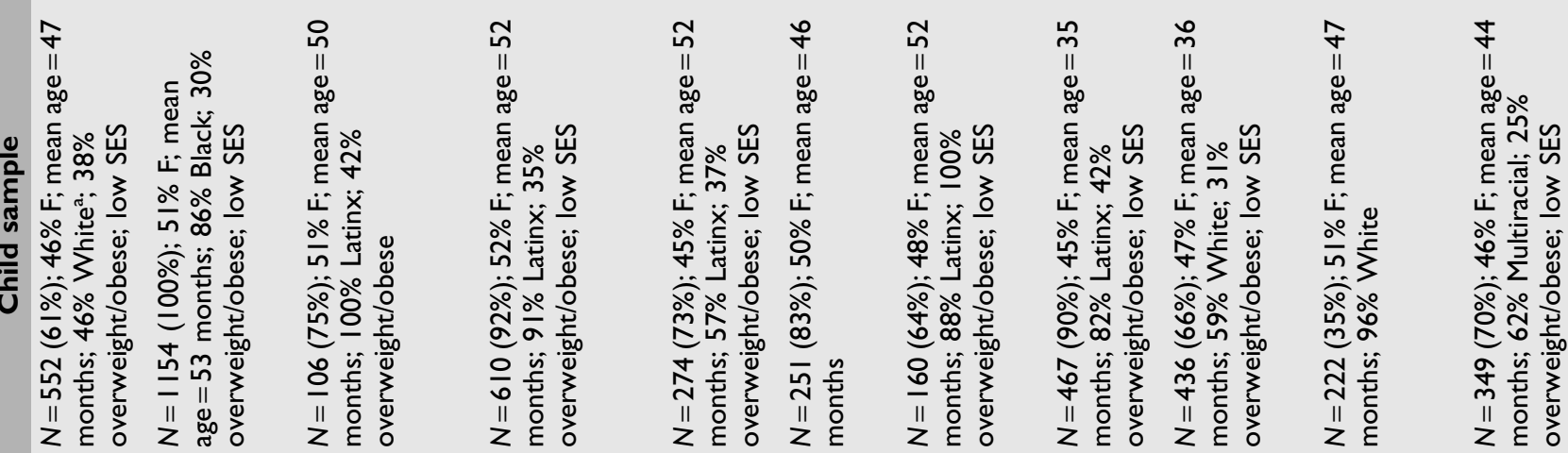

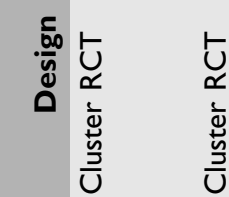

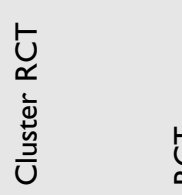

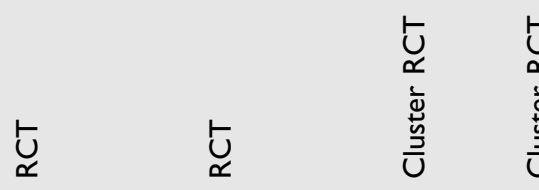

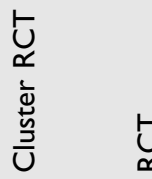

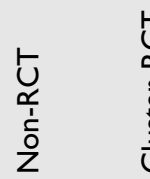

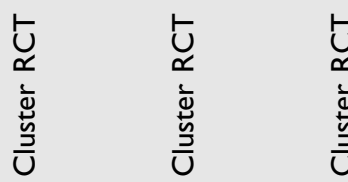

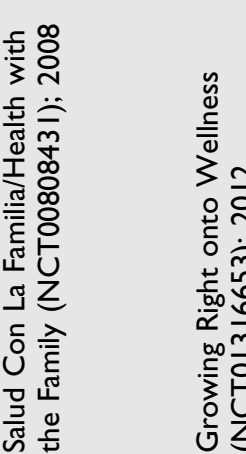

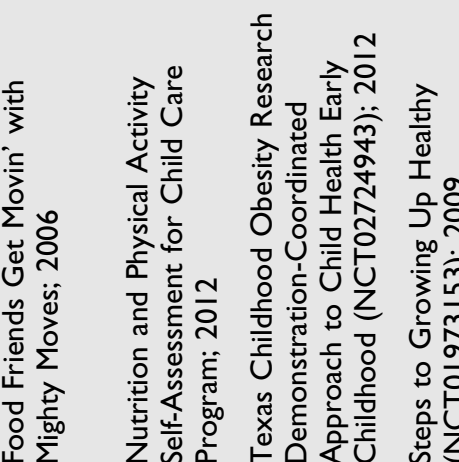

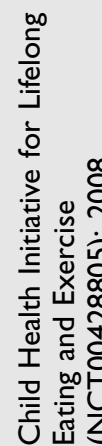

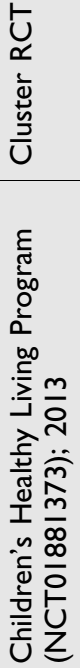




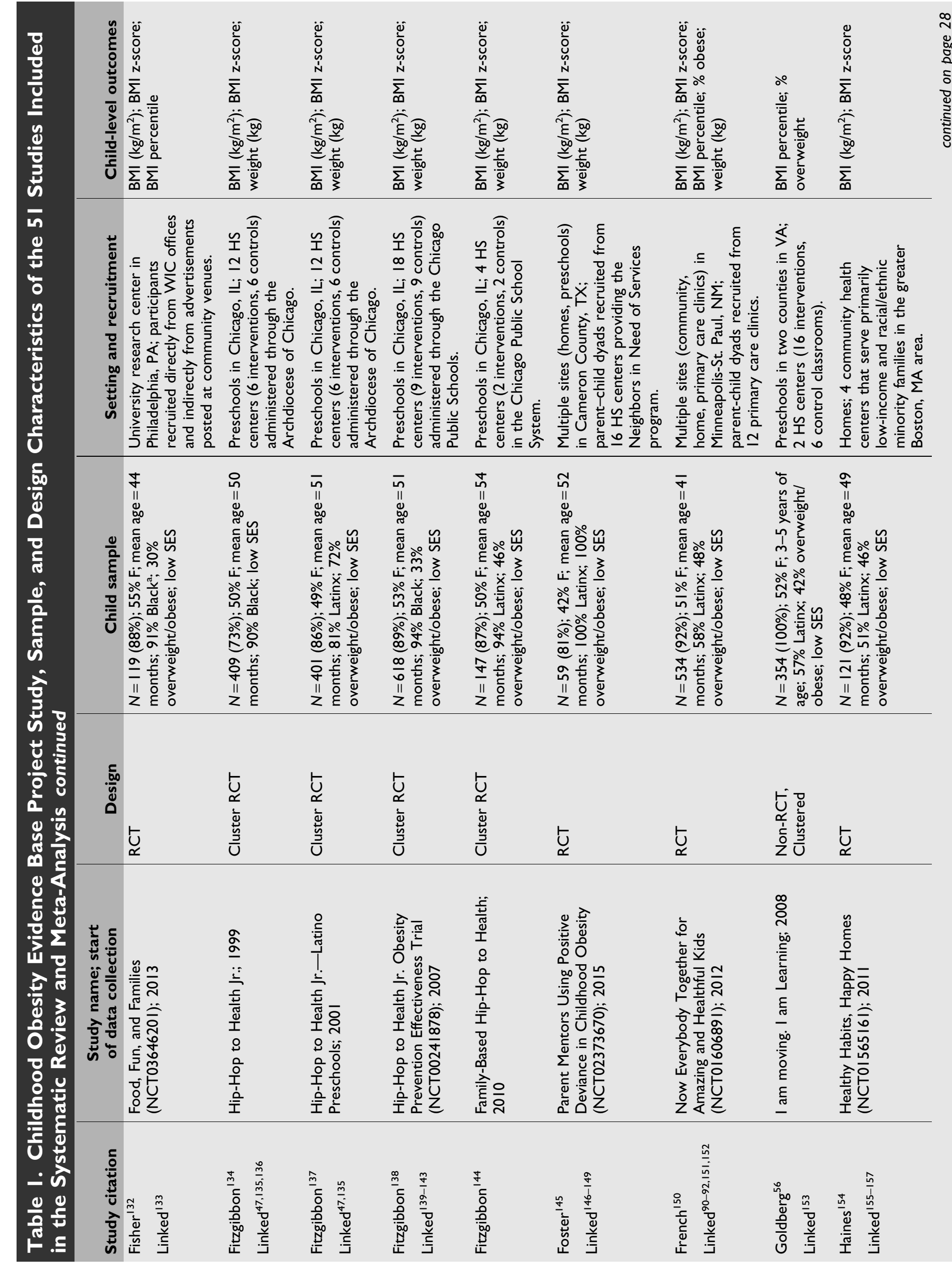




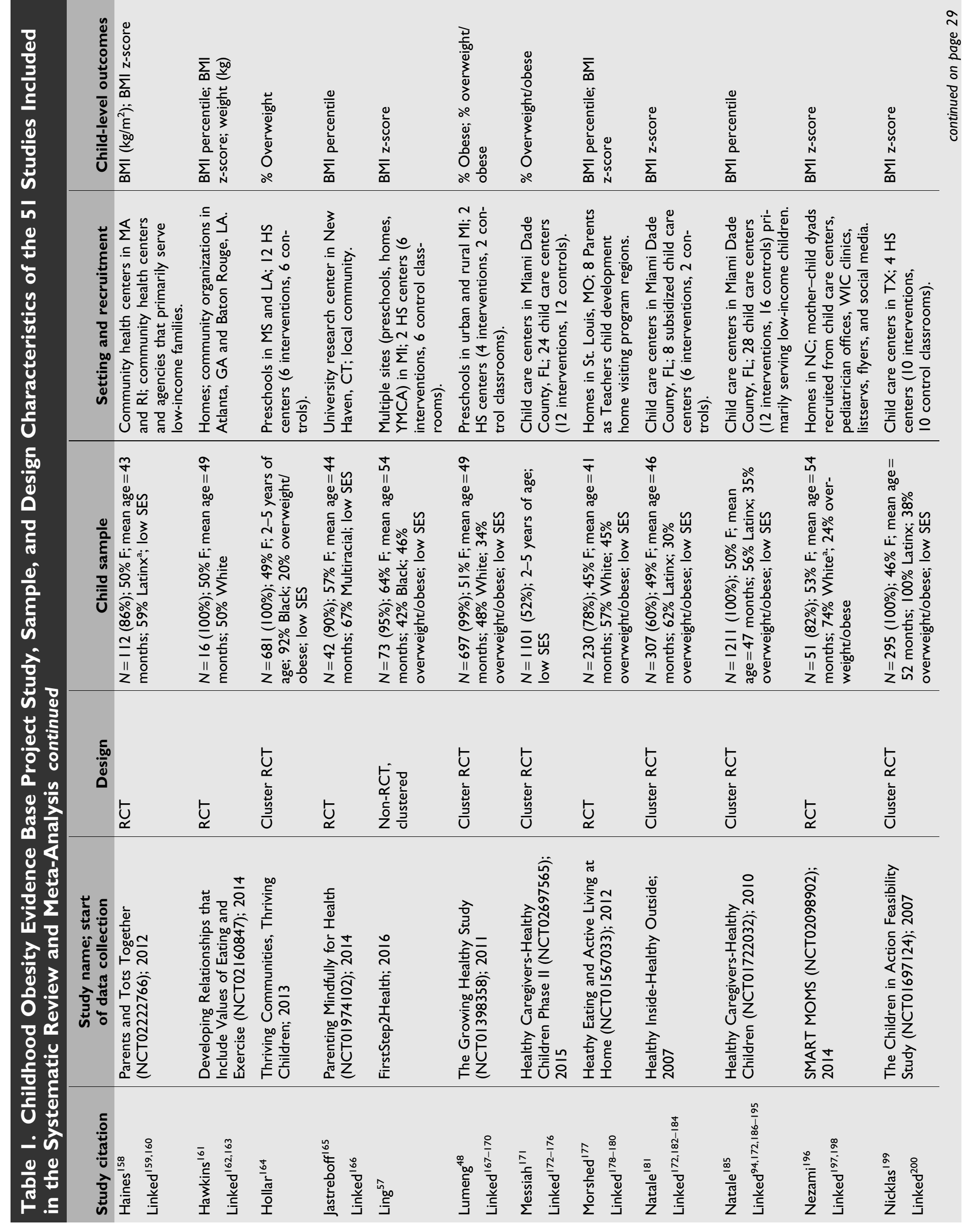




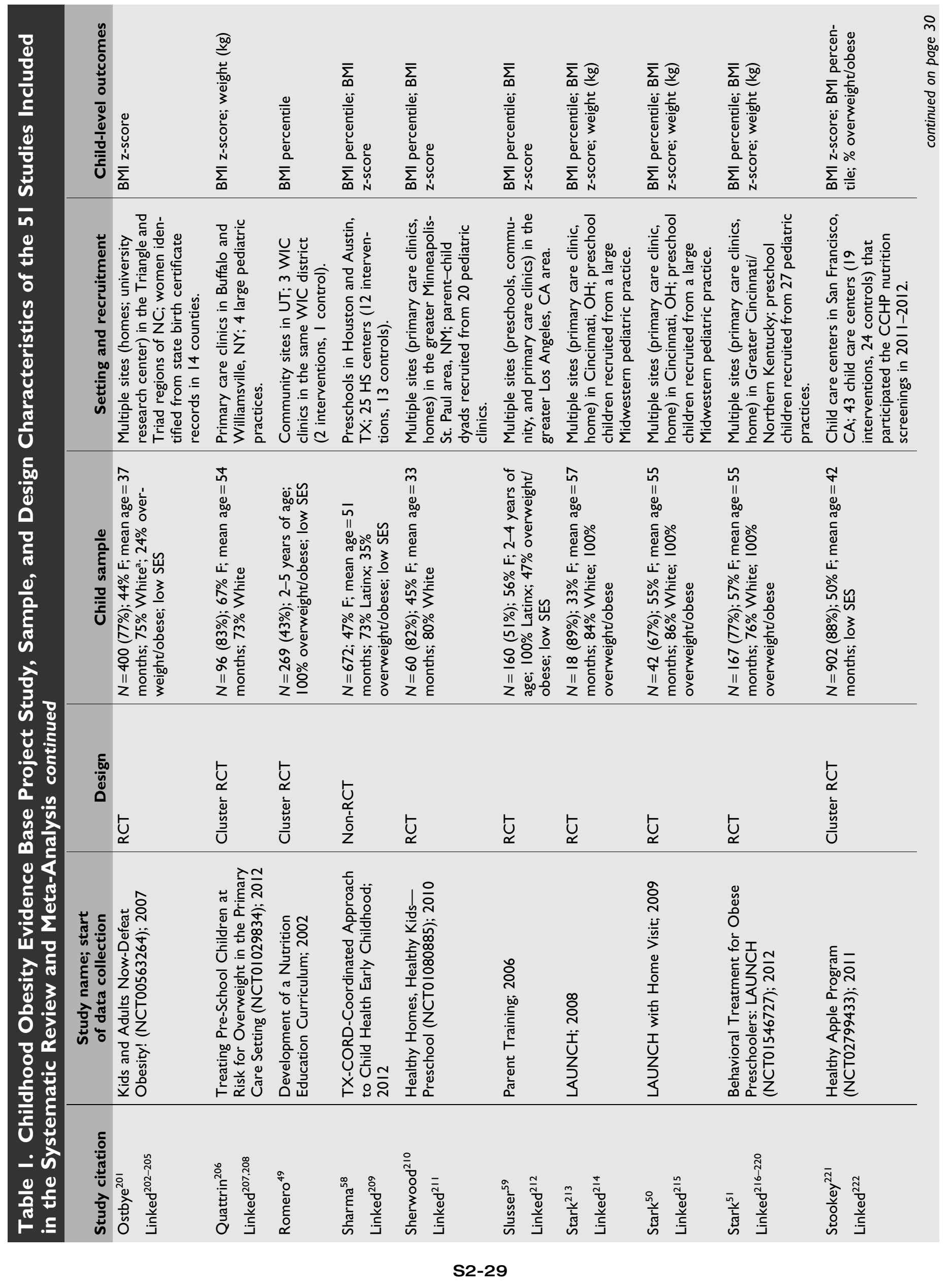




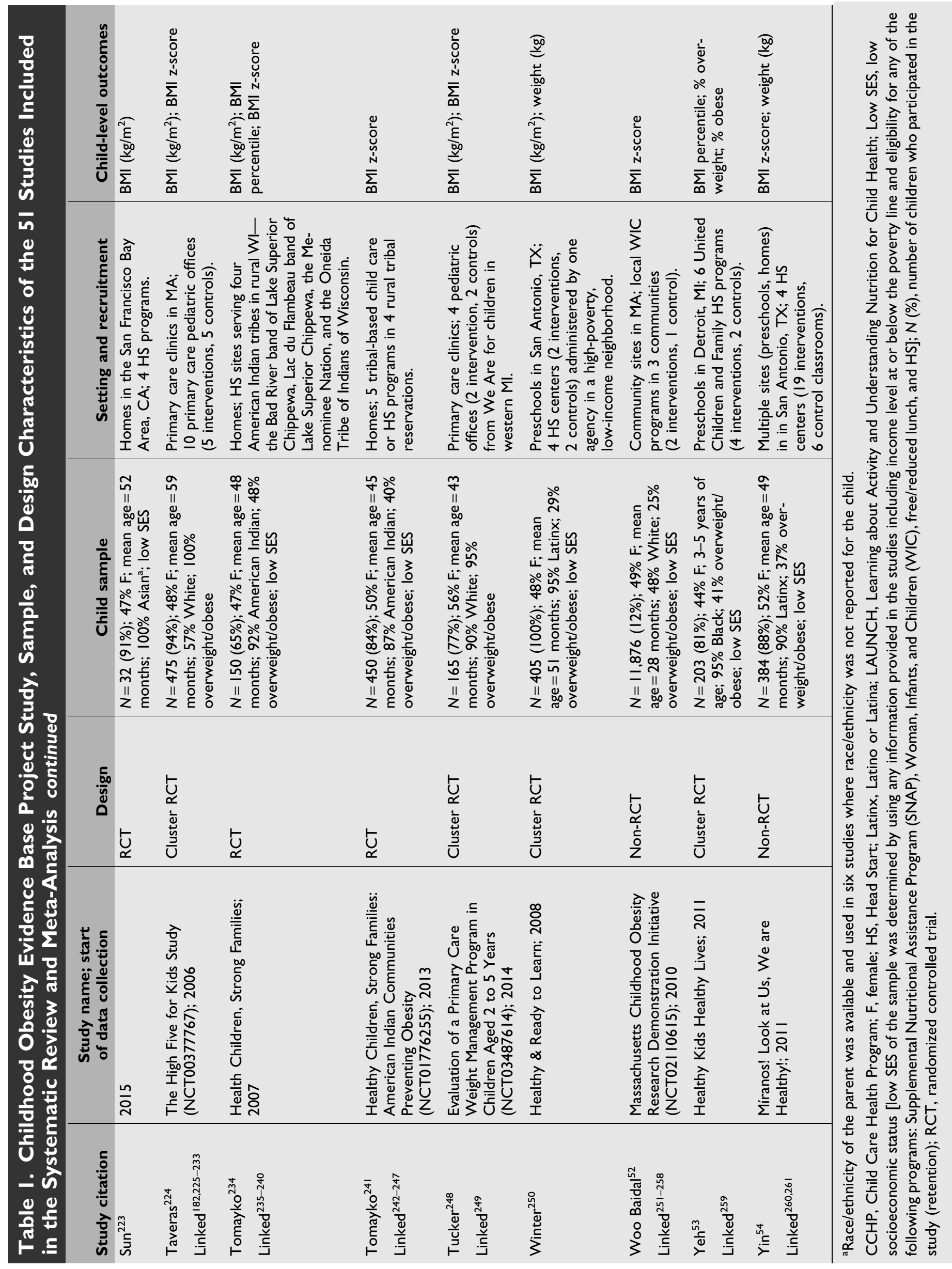


Table 2. Childhood Obesity Evidence Base

Project Study, Sample, and Intervention

Characteristics of the 5 I Studies

(58 Interventions) Included in the Childhood Obesity Prevention Meta-Analysis

\begin{tabular}{|c|c|}
\hline Study and design characteristics & \\
\hline Publication year, median (range) & 2015 (2005-2019) \\
\hline Data collection year, median (range) & $2010(1995-2016)$ \\
\hline Source, $n(\%)$ & \\
\hline Journal & $48(94)$ \\
\hline Dissertation & $2(4)$ \\
\hline Conference Abstract & I (2) \\
\hline Funded research, \% & 98 \\
\hline RCT, \% & 88 \\
\hline U.S. region, \% & \\
\hline Northeast & 18 \\
\hline Southeast & 24 \\
\hline Midwest & 29 \\
\hline West & 10 \\
\hline Southwest & 14 \\
\hline Multiple regions & 6 \\
\hline Setting, \%, $(n=27)$ & \\
\hline Urban & 70 \\
\hline Rural & II \\
\hline Urban and suburban & 4 \\
\hline Urban and rural & 15 \\
\hline Recruitment site, \% & \\
\hline Child care/preschool & 55 \\
\hline Clinical contact & 22 \\
\hline Community/other & 14 \\
\hline Multiple & 10 \\
\hline Random assignment, \% & \\
\hline Quasi-experimental & 12 \\
\hline Random assignment of groups & 49 \\
\hline Matching then random assignment & 16 \\
\hline True randomization & 24 \\
\hline Multisite study design, \% & 67 \\
\hline No. of post-tests, median (range) & I (I-5) \\
\hline Sample characteristics & \\
\hline Children & \\
\hline Sample size & 29,085 \\
\hline Retention, M \% (SD), $(n=50)$ & $80(18)$ \\
\hline Girls, M \% (SD), $(n=49)$ & $50(5)$ \\
\hline
\end{tabular}

Table 2. Childhood Obesity Evidence Base

Project Study, Sample, and Intervention

Characteristics of the 5 I Studies

(58 Interventions) Included in the Childhood

Obesity Prevention Meta-Analysis continued
7I (3I)

39 (76)

Low SES, $n$ (\%)

Program eligibility, \%

Free/reduced lunch

8

SNAP or WIC

35

HS

45

Medicaid

12

48 (7)

6 (12)

18 (35)

4 (8)

2 (4)

20 (39)

I (2)

Child BMI status, $M$ \% (SD)

Underweight $(n=39)$

I (2)

Normal weight $(n=38)$

47 (27)

Overweight $(n=40)$

20 (12)

Obese $(n=40)$

29 (27)

Parent/caregivers

Sample size $(n=35)$

Female, M \% (SD), $(n=27)$

10,410

94 (8)

Age in years, $M(S D),(n=25)$

32 (2)

Non-white, $M$ \% (SD)

74 (30)

Marital status, $M$ \% (SD)

Married/cohabitating $(n=20)$

64 (23)

Divorced/widowed $(n=5)$

Single $(n=10)$

36 (29)

Education, $M$ \% (SD)

Less than high school $(n=15)$

3 I (20)

High school/GED $(n=13)$

Some college/technical $(n=14)$

College degree or higher $(n=18)$

32 (28)

Employment, M \% (SD)

Full-time $(n=10)$

Part-time $(n=8)$
$18(8)$ 
Table 2. Childhood Obesity Evidence Base Project Study, Sample, and Intervention Characteristics of the 5 I Studies

(58 Interventions) Included in the Childhood Obesity Prevention Meta-Analysis continued

\begin{tabular}{|c|}
\hline Unemployed $(n=10)$ \\
\hline Student $(n=5)$ \\
\hline Disabled $(n=5)$ \\
\hline Language, $M \%$ (SD) \\
\hline English $(n=13)$ \\
\hline Non-English $(n=14)$ \\
\hline English+other language $(n=13)$ \\
\hline BMI status, $\mathrm{M} \%(\mathrm{SD})$ \\
\hline Underweight $(n=18)$ \\
\hline Normal weight $(n=18)$ \\
\hline Overweight $(n=14)$ \\
\hline Obesity $(n=14)$ \\
\hline Facilitator $^{\mathrm{a}}$ characteristics \\
\hline Facilitators for children \\
\hline $\begin{array}{l}\text { Facilitators, median (range), } \\
(n=36)\end{array}$ \\
\hline Type of facilitators, $\%,(n=45)$ \\
\hline Not reported \\
\hline No facilitators \\
\hline $\begin{array}{l}\text { Preschool/child care teachers, } \\
\text { assistants, or staff }\end{array}$ \\
\hline Research staff (BA/MA) \\
\hline Parent peers \\
\hline Paraprofessional \\
\hline Research Staff $(B A / M A)+P a r a$ \\
\hline Research Staff (BA/MA)+Prof \\
\hline Para+Prof \\
\hline Multiple \\
\hline Language, M \% (SD), $(n=13)$ \\
\hline English \\
\hline Non-English \\
\hline English+other language \\
\hline Facilitators for parents/caregivers \\
\hline $\begin{array}{l}\text { Facilitators, median (range), } \\
(n=36)\end{array}$ \\
\hline Type of facilitators, $\%,(n=46)$ \\
\hline Not reported \\
\hline No facilitators \\
\hline
\end{tabular}

54 (42)

38 (5I)

$0(0)$

$62(5 I)$

I (0-5)
Table 2. Childhood Obesity Evidence Base Project Study, Sample, and Intervention Characteristics of the 5 I Studies

(58 Interventions) Included in the Childhood Obesity Prevention Meta-Analysis continued

42 (18) Preschool/child care teachers,

9

assistants, or staff

Research staff (BA/MA)

Parent peers

Paraprofessional

Professional

Research staff (BA/MA)+Para

Research staff (BA/MA)+Prof

Para+Prof

Multiple

Language, $M$ \% (SD), $(n=12)$

English

Non-English

English+other language

Facilitators for facilitators

Facilitators, median (range), $(n=10)$

Type of facilitators, \%, $(n=39)$

Not reported

Paraprofessional

Professional

Para+Prof

Language, $M \%(n=2)$

English

Non-English

English+other language

Intervention characteristics

Theory/model

$\%$ SCT/SLT

SEM

Other

Multiple

40

31

23

6

0

0

100

51

9

16

24

Delivery setting, \%

Child care/preschool

4I

Clinical practice

10

Community

12

Home

12

continued

4


Table 2. Childhood Obesity Evidence Base Project Study, Sample, and Intervention Characteristics of the 5 I Studies

(58 Interventions) Included in the Childhood Obesity Prevention Meta-Analysis continued

Multiple/other
Recipient, \%
Child
Parent/caregiver
Child care provider
Physician/health care provider
Content focus, \%
Diet
Physical Activity
Diet and Physical Activity
Multicomponent/Other
Duration (months), median (range)
Child
No. of sessions, median (range), $k=42$
Total dose (in minutes), median, $k=30$
Parent
No. of sessions, median (range), $k=46$
Total dose (in minutes), median, $k=30$
No sessions, median (range), $k=27$
Pates), median, $k=22$

Controls

Type of control, \%

Wait-list

No treatment

Education only

Irrelevant content, matched

Irrelevant content, not matched

Relevant content, matched

Relevant content, not matched

Active controls, \% $(n=5 \mathrm{I})$
The mean number of components identified across the interventions was $20(\mathrm{SD}=6$, range $=7-34)$. The 5 most commonly reported components across the 58 interventions included were: (1) used research-based approaches or curriculum $(84 \%)$; (2) provided written resources to caregivers $(60 \%)$; (3) provided initial or one-time training opportunities for facilitators $(59 \%)$; (4) provided education about nutrition and healthy eating patterns to caregivers (53\%); and (5) provided curricular materials to facilitators $(50 \%)$. (A list of the intervention components can be found in Table 3.)

\section{Synthesis of Results}

All studies assessed BMI at the end of treatment except for one study, ${ }^{59}$ which did not include an immediate post-test; sixteen studies assessed BMI at a longer-follow-up (median $=52$ weeks, range $=18-143$ weeks). Hedge's $g$ (and homogeneity statistics) for each BMI outcome by assessment interval is presented in Supplementary Table S3. Analyses indicated that children in the intervention group had a smaller BMI increase at the immediate post-test $(g=0.10,95 \%$ $\mathrm{CI}=0.02-0.18 ; k=55 ; 0$ weeks $)$ and at the last follow-up $(g=0.17,95 \% \mathrm{CI}=0.04-0.30 ; k=14 ;$ range $=18-143$ weeks $)$ relative to the comparison group.* [Extreme outliers were detected at both the immediate post-test ( 2 out of $57 \mathrm{ES}$ ) and last follow-up (2 out of $16 \mathrm{ES}$ ) assessment and were removed from the analyses; see Supplementary Table S3.] The hypothesis of homogeneity was supported for BMI [immediate post-test: $Q(54)=67.08, p=0.109 ; I^{2}=20,95 \% \mathrm{CI}=0-43$; last follow-up: $Q(13)=11.91, \quad p=0.535 ; \quad I^{2}=0,95 \%$ $\mathrm{CI}=0-74]$, but uncertainty limits were wide at the last follow-up and exceeded the 50\% threshold. (Forest plots of the overall BMI at the immediate post-test and last followup are found in Figs. 2 and 3.)

When pooling the separate BMI metrics, analyses showed that children in the intervention group had a lower BMI percentile at the immediate post-test $(g=0.15,95 \%$ $\mathrm{CI}=0.04-0.26 ; k=19)$ and last follow-up $(g=0.59,95 \%$ $\mathrm{CI}=0.14-1.13 ; k=6)$ and a lower BMI $z$-score at last follow-up $(g=0.20,95 \% \mathrm{CI}=0.06-0.34 ; k=12)$ relative to the comparison group. Intervention children also had a lower weight at the immediate post-test $(g=0.35,95 \%$ $\mathrm{CI}=0.16-0.55 ; k=16)$ and last follow-up $(g=0.26,95 \%$ $\mathrm{CI}=0.05-0.48 ; k=11)$ assessment compared with the comparison group. The hypothesis of homogeneity was not supported for weight [immediate post-test: $Q(15)=109.93$, $p<0.001 ; \quad I^{2}=86, \quad 95 \% \quad \mathrm{CI}=79-90 ;$ last follow-up: $\left.Q(11)=45.06, p<0.001 ; I^{2}=78,95 \% \mathrm{CI}=61-88\right]$, and uncertainty limits exceeded the $75 \%$ threshold.

*Sensitivity analyses showed that excluding studies in which multiple interventions were compared with a single control condition did not change the magnitude or direction of the findings at the immediate post-test ( $g=0.10,95 \% \mathrm{Cl}=0.07-0.13 ; k=42 ; 0$ weeks) but weakened the findings at the last follow-up $(g=0.13,95 \% \mathrm{Cl}=-0.0 \mathrm{I}-0.27 ; k=10$; range $=18-143$ weeks).

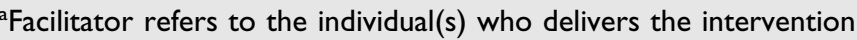
content (e.g., research assistant, teacher, physician) to the children, caregiver, or facilitator.

$\mathrm{BA} / \mathrm{MA}$, bachelor or master's degree; $k$, number of interventions; $M$, mean; matched, for time or contact; $n$, number of studies reporting on the specified characteristic; Para, paraprofessional; Prof, professional; SCT/SLT, Social Cognitive Theory/Social Learning Theory; SD, standard deviation; SEM, socioecological model. 


\section{Table 3. Childhood Obesity Evidence Base Project Taxonomy of Components Identified, Frequency, and Impact on the Overall BMI across the 58 Interventions Included in the Analyses}

\begin{tabular}{|c|c|c|c|c|c|}
\hline \multirow[b]{3}{*}{ Intervention components } & \multirow[b]{3}{*}{$\%$} & \multicolumn{4}{|c|}{ Impact on BMI } \\
\hline & & \multicolumn{2}{|c|}{ Immediate } & \multicolumn{2}{|c|}{ Final } \\
\hline & & B (SE) & $p$ & B (SE) & $p$ \\
\hline Activities to support behavior change & & $0.03(0.01)$ & 0.024 & $0.01(0.02)$ & 0.743 \\
\hline Implement structures of accountability & 28 & $0.06(0.04)$ & 0.117 & $0.09(0.09)$ & 0.334 \\
\hline Incorporate implementation of self-reflection strategies & 19 & $0.02(0.05)$ & 0.678 & $-0.03(0.08)$ & 0.723 \\
\hline Implement media campaigns & 2 & $0.18(0.11)$ & 0.098 & $-0.03(0.10)$ & 0.781 \\
\hline Incorporate financial incentives & 19 & $0.03(0.04)$ & 0.446 & $0.03(0.08)$ & 0.663 \\
\hline Engage caregivers in praise/encouragement for positive behavior & 22 & $0.09(0.05)$ & 0.049 & $0.34(0.19)$ & 0.092 \\
\hline Engage caregivers in goal setting & 31 & $0.07(0.04)$ & 0.122 & $-0.03(0.08)$ & 0.689 \\
\hline Engage caregivers to serve as role models for children & 19 & $0.05(0.04)$ & 0.234 & - & - \\
\hline Engage facilitators in praise/encouragement for positive behavior & 9 & $-0.03(0.05)$ & 0.476 & - & - \\
\hline Instructional strategies & & $-0.01(0.01)$ & 0.286 & $-0.01(0.02)$ & 0.486 \\
\hline Provide toys/books/games/stickers for child engagement & 34 & $-0.04(0.04)$ & 0.308 & $-0.11(0.08)$ & 0.187 \\
\hline Utilize arts and music & 24 & $-0.01(0.04)$ & 0.840 & $-0.06(0.07)$ & 0.421 \\
\hline Utilize games, imaginative play, or storytelling & 29 & $0.01(0.04)$ & 0.809 & $-0.08(0.07)$ & 0.311 \\
\hline Utilize a stepped-intensity approach & 14 & $-0.03(0.07)$ & 0.732 & $0.08(0.08)$ & 0.381 \\
\hline Utilize written activities & 10 & $-0.00(0.05)$ & 0.924 & $-0.04(0.08)$ & 0.626 \\
\hline Utilize modeling/demonstration & 22 & $0.06(0.04)$ & 0.116 & $0.12(0.12)$ & 0.342 \\
\hline Utilize media for instruction & 16 & $-0.07(0.06)$ & 0.287 & $-0.08(0.11)$ & 0.452 \\
\hline Utilize hands-on approach & 34 & $-0.06(0.03)$ & 0.069 & $-0.11(0.08)$ & 0.203 \\
\hline Utilize reflective listening & 7 & $-0.00(0.07)$ & 0.988 & $-0.02(0.09)$ & 0.860 \\
\hline Utilize discussion & 24 & $-0.05(0.04)$ & 0.207 & $-0.01(0.08)$ & 0.895 \\
\hline Utilize role-playing for instruction & 12 & $-0.05(0.06)$ & 0.428 & $0.04(0.13)$ & 0.782 \\
\hline Utilize group instruction & 41 & $-0.03(0.03)$ & 0.354 & $0.09(0.08)$ & 0.248 \\
\hline Utilize telephone calls & 19 & $0.06(0.05)$ & 0.187 & $-0.03(0.08)$ & 0.689 \\
\hline Utilize dual language instruction/materials & 26 & $-0.06(0.03)$ & 0.100 & $0.02(0.07)$ & 0.818 \\
\hline Utilize field trips/site visits & 16 & $-0.02(0.06)$ & 0.729 & $-0.02(0.09)$ & 0.860 \\
\hline Activities for supporting caregivers & & $0.01(0.01)$ & 0.447 & $0.00(0.01)$ & 0.914 \\
\hline Engage experts to provide technical assistance to caregivers & 16 & $0.00(0.05)$ & 0.980 & - & - \\
\hline Provide audiovisual media resources to caregivers & 5 & $-0.11(0.12)$ & 0.366 & $-0.12(0.16)$ & 0.465 \\
\hline Provide materials to support healthy eating patterns to caregivers & 28 & $0.02(0.04)$ & 0.710 & $0.05(0.08)$ & 0.532 \\
\hline Provide materials to support screen time reduction to caregivers & 14 & $0.04(0.05)$ & 0.441 & $-0.03(0.08)$ & 0.723 \\
\hline Provide written resources to caregivers & 60 & $-0.05(0.04)$ & 0.170 & $-0.09(0.09)$ & 0.331 \\
\hline Provide access to social media platforms/websites to caregivers & 10 & $-0.01(0.06)$ & 0.873 & $-0.05(0.08)$ & 0.537 \\
\hline Provide education about obesity risk/awareness to caregivers & 10 & $-0.07(0.06)$ & 0.268 & - & - \\
\hline Provide education about physical activity to caregivers & 38 & $0.06(0.04)$ & 0.148 & $0.01(0.08)$ & 0.858 \\
\hline Provide education about healthy sleep patterns to caregivers & 12 & $-0.08(0.06)$ & 0.211 & $-0.01(0.09)$ & 0.938 \\
\hline Provide education about the importance of routines to caregivers & 14 & $-0.04(0.08)$ & 0.603 & $0.15(0.15)$ & 0.338 \\
\hline & & & & continued o & age 35 \\
\hline
\end{tabular}


Table 3. Childhood Obesity Evidence Base Project Taxonomy of Components Identified, Frequency, and Impact on the Overall BMI across the 58 Interventions Included in the Analyses continued

\section{Intervention components}

Provide education about nutrition and heathy eating patterns to caregivers

Provide education about child behavior management to caregivers

Provide education about modeling healthy behaviors to caregivers

Provide education about sources of stress and coping strategies to caregivers

Provide education about social and emotional skills to caregiver

Provide education about the importance of built environment to caregiver

Provide education about health and wellness content to caregivers

Include activities to promote problem-solving to caregivers

Provide education about the importance of screen time reduction to caregivers

Implement personalized support for caregiver

Implement follow-up support for caregivers

Implement support groups for caregivers

Provide materials to support self-control in children

Facilitator training activities

Use a train-the-trainer model

Provide curricular materials to facilitators

Provide regular training opportunities for facilitators

Provide initial or one-time training opportunities for facilitators

Provide physical activity education and training to facilitators

Provide healthy eating education and training to facilitators

Provide food preparation education and training to facilitators

Provide nutrition education and training to facilitators

Engagement of facilitators

Engage pediatricians/health care providers

Engage child care providers

Engage families

Engage community organizations

Policy-based strategies

Implement nutrition standards

Implement earned income tax credit

Implement policies regulating food/beverage costs

Implement policies for regulation of food/beverage access

Implement SNAP/WIC policies

Implement policies for increasing physical activity

\section{Impact on BMI}

\begin{tabular}{|c|c|c|c|c|}
\hline \multirow[b]{2}{*}{$\%$} & \multicolumn{2}{|c|}{ Immediate } & \multicolumn{2}{|c|}{ Final } \\
\hline & B (SE) & $p$ & B (SE) & $p$ \\
\hline 53 & $0.01(0.04)$ & 0.861 & $-0.05(0.08)$ & 0.545 \\
\hline I & $0.08(0.05)$ & 0.092 & $0.06(0.08)$ & 0.437 \\
\hline 6 & $0.00(0.04)$ & 0.971 & $-0.05(0.09)$ & 0.563 \\
\hline 9 & $-0.11(0.08)$ & 0.167 & $0.04(0.19)$ & 0.849 \\
\hline 2 & $0.06(0.05)$ & 0.228 & - & - \\
\hline 4 & $0.05(0.05)$ & 0.328 & $0.01(0.09)$ & 0.947 \\
\hline 10 & $-0.01(0.05)$ & 0.920 & $-0.02(0.09)$ & 0.860 \\
\hline 17 & $0.02(0.07)$ & 0.824 & $0.23(0.12)$ & 0.074 \\
\hline 31 & $0.13(0.04)$ & 0.002 & $0.10(0.08)$ & 0.267 \\
\hline 29 & $0.05(0.04)$ & 0.235 & $0.00(0.08)$ & 0.992 \\
\hline 21 & $-0.00(0.05)$ & 0.928 & $-0.08(0.13)$ & 0.566 \\
\hline 2 & $0.01(0.27)$ & 0.972 & - & - \\
\hline 0 & $\begin{array}{c}- \\
-0.01(0.01)\end{array}$ & $\begin{array}{c}- \\
0.575\end{array}$ & $\begin{array}{c}- \\
-0.12(0.06)\end{array}$ & $\frac{-}{0.091}$ \\
\hline 22 & $0.02(0.04)$ & 0.620 & $-0.02(0.09)$ & 0.860 \\
\hline 50 & $-0.02(0.04)$ & 0.650 & $0.01(0.07)$ & 0.929 \\
\hline & $-0.01(0.04)$ & 0.738 & $-0.12(0.09)$ & 0.860 \\
\hline 59 & $-0.01(0.04)$ & 0.831 & $-0.05(0.08)$ & 0.488 \\
\hline 21 & $-0.03(0.03)$ & 0.334 & - & - \\
\hline 7 & $-0.00(0.06)$ & 0.994 & - & - \\
\hline 3 & $-0.12(0.16)$ & 0.434 & - & - \\
\hline 17 & $-0.03(0.04)$ & 0.493 & $-0.11(0.17)$ & 0.540 \\
\hline & $0.02(0.02)$ & 0.213 & $-0.01(0.06)$ & 0.893 \\
\hline 17 & $0.11(0.04)$ & 0.012 & $-0.05(0.87)$ & 0.541 \\
\hline 14 & $0.07(0.04)$ & 0.076 & $-0.00(0.12)$ & 0.975 \\
\hline 29 & $-0.01(0.04)$ & 0.819 & $0.04(0.19)$ & 0.849 \\
\hline 22 & $-0.03(0.04)$ & 0.442 & $-0.01(0.08)$ & 0.864 \\
\hline & $-0.01(0.01)$ & 0.196 & - & - \\
\hline 19 & $-0.04(0.03)$ & 0.220 & - & - \\
\hline 0 & - & - & - & - \\
\hline 0 & - & - & - & - \\
\hline 10 & $-0.03(0.04)$ & 0.414 & - & - \\
\hline 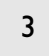 & $-0.06(0.04)$ & 0.159 & - & - \\
\hline 10 & $-0.04(0.04)$ & 0.344 & - & - \\
\hline
\end{tabular}

continued on page 36 
Table 3. Childhood Obesity Evidence Base Project Taxonomy of Components Identified, Frequency, and Impact on the Overall BMI across the 58 Interventions Included in the Analyses continued

Intervention components

Activities related to physical activity/environment

Focus on physical activity education

Focus on importance of reduced screen time

Provide materials/space to support physical activity to caregivers

Provide materials/space to support physical activity to facilitators

Engage caregivers in supporting physical activities

Engage child care providers in supporting physical activity

Implement reduction of sedentary behaviors

Implement "fun" physical activities to engage children

Include structured physical activities

Include free play

Activities related to food/food environment

Focus on food preparation education

Focus on nutrition-related education

Provide food to encourage healthy eating to children

Provide healthy recipes/shopping lists/menus to caregivers

Engage child care providers in facilitating healthy eating patterns

Decrease less healthy food options

Increase healthy food options

Include opportunities for children to prepare foods

Include opportunities for children to try new foods

Engage caregivers in facilitating healthy eating patterns

Characteristics of the intervention

Utilized recognized standards or recommendations

Utilized research-based approaches or curriculum

Used a multi-level approach

Used culturally tailored intervention

Documentation of intervention implementation/quality

Engage caregiver/family in intervention development

Engage pediatricians/health care providers in intervention development

Included home-visits
Engage child care providers in intervention development

Impact on BMI

\begin{tabular}{|c|c|c|c|}
\hline \multicolumn{2}{|c|}{ Immediate } & \multicolumn{2}{|c|}{ Final } \\
\hline B (SE) & $p$ & B (SE) & $p$ \\
\hline$-0.00(0.01)$ & 0.946 & $-0.03(0.03)$ & 0.350 \\
\hline$-0.02(0.04)$ & 0.609 & $-0.04(0.10)$ & 0.695 \\
\hline$-0.03(0.04)$ & 0.431 & $-0.08(0.08)$ & 0.321 \\
\hline$-0.05(0.04)$ & 0.231 & $0.04(0.19)$ & 0.849 \\
\hline$-0.03(0.05)$ & 0.572 & $-0.16(0.18)$ & 0.393 \\
\hline $0.07(0.04)$ & 0.067 & $0.03(0.08)$ & 0.707 \\
\hline $0.03(0.04)$ & 0.428 & - & - \\
\hline$-0.03(0.04)$ & 0.465 & $-0.10(0.17)$ & 0.540 \\
\hline $0.02(0.04)$ & 0.568 & $-0.05(0.07)$ & 0.501 \\
\hline $0.01(0.04)$ & 0.841 & $0.00(0.08)$ & 0.996 \\
\hline $0.02(0.06)$ & 0.683 & - & - \\
\hline $0.01(0.01)$ & 0.383 & $0.00(0.03)$ & 0.965 \\
\hline $0.06(0.05)$ & 0.256 & $-0.10(0.09)$ & 0.264 \\
\hline $0.01(0.04)$ & 0.851 & $0.02(0.07)$ & 0.830 \\
\hline$-0.04(0.04)$ & 0.247 & $-0.12(0.07)$ & 0.139 \\
\hline $0.07(0.05)$ & 0.115 & $-0.02(0.10)$ & 0.781 \\
\hline $0.01(0.05)$ & 0.746 & $0.19(0.11)$ & 0.108 \\
\hline $0.02(0.04)$ & 0.519 & $-0.05(0.08)$ & 0.511 \\
\hline $0.02(0.04)$ & 0.623 & $-0.02(0.09)$ & 0.860 \\
\hline$-0.03(0.07)$ & 0.605 & $-0.02(0.09)$ & 0.860 \\
\hline $0.02(0.04)$ & 0.695 & $0.15(0.08)$ & 0.097 \\
\hline $0.04(0.04)$ & 0.414 & $0.06(0.08)$ & 0.415 \\
\hline $0.00(0.01)$ & 0.982 & $0.02(0.02)$ & 0.296 \\
\hline$-0.03(0.03)$ & 0.426 & $0.10(0.07)$ & 0.192 \\
\hline$-0.00(0.06)$ & 0.945 & - & - \\
\hline$-0.01(0.04)$ & 0.676 & $-0.03(0.10)$ & 0.781 \\
\hline$-0.00(0.04)$ & 0.969 & $0.02(0.08)$ & 0.791 \\
\hline $0.03(0.04)$ & 0.387 & $0.14(0.15)$ & 0.351 \\
\hline $0.02(0.05)$ & 0.766 & $0.01(0.08)$ & 0.902 \\
\hline$-0.03(0.04)$ & 0.524 & $0.06(0.09)$ & 0.493 \\
\hline $0.02(0.06)$ & 0.786 & $0.06(0.09)$ & 0.493 \\
\hline $0.07(0.07)$ & 0.322 & $0.14(0.15)$ & 0.351 \\
\hline
\end{tabular}

The overall frequency is the proportion of interventions that included the specific component expressed as a percentage. Bold values indicates significant moderator of BMI at the immediate or final post-intervention assessment. Dashes indicate that meta-regression could not be performed due to insufficient cases or when the variable was dropped due to multicollinearity.

SE, standard error. 


\section{Synthesis of Moderator Analyses}

Meta-regression (using restricted maximum likelihood to estimate the between-study variance) was conducted to examine proposed moderators of BMI (see Moderator Analyses). Interventions that sampled fewer children of low socioeconomic status $(\beta=-0.20, p=0.002)$, sampled more children meeting criteria for overweight or obesity $(\beta=0.23, \quad p=0.009)$, recruited samples from clinical practices $(\beta=0.20, p<0.001 ;$ e.g., pediatrician offices, primary care), and implemented the intervention in a clinical practice $(\beta=0.24, p<0.001)$ were more likely to reduce children's BMI at the immediate post-test.

We also examined whether components of the intervention impacted BMI. Overall, children had a lower BMI at the immediate follow-up when the intervention delivered more, rather than fewer, activities supporting behavior change, $\beta=0.03, p=0.024$ (total components, see Table 3). Interventions that: (1) engaged caregivers in praise/encouragement for positive health-related behavior $(\beta=0.09, p=0.049)$; (2) provided education about the importance of screen time reduction to caregivers $(\beta=0.13, p=0.002)$; and (3) engaged pediatricians/health care providers in delivering intervention content $(\beta=0.11, p=0.012)$ were more successful than interventions that did not include these components in reducing BMI in children at the immediate post-test.

When the intervention components were simultaneously entered in a multiple meta-regression model, only providing education about the importance of screen time reduction to caregivers emerged as a significant moderator of BMI at the end of the intervention. The model was significant $\left[F(3,51)=4.33, p=0.009, I^{2}\right.$ residual $\left.=5 \%\right]$ and accounted for $89 \%$ of the variance in the change in BMI. Adjusting for multiple testing using the permutation test (5000 permutations) confirmed that interventions providing education about the importance of screen time reduction to caregivers differ on average from interventions that did not include this component (adjusted $p$-value $=0.048$ ) ${ }^{\dagger}$

\footnotetext{
We did not include other significant sample or intervention features (Supplementary Table S4) in this model due to multicollinearity and overfitting, but we conducted exploratory analyses to evaluate the significant univariate moderators, controlling for screen time reduction, in separate multiple meta-regression models. Our base model assessed screen time reduction only $\left[F(1,53)=10.76, p=0.002 ; I^{2}\right.$ residual $=5 \% ; \tau^{2}=0.0003$ ] which was compared with models that also included the significant univariate moderator (model 2). Only one pattern emerged: The model including socioeconomic status provided a better fit to the data, indicating that childhood obesity interventions that sampled fewer children of low socioeconomic status $(\beta=-0.14$, $p=0.043)$, and provided education on screen time reduction $(\beta=0.09$, $p=0.038)$, were more likely to reduce children's BMI at the immediate assessment $\left[F(2,52)=7.72, p=0.00 \mathrm{I} ; I^{2}\right.$ residual $\left.=0 \% ; \tau^{2}=0.0002\right)$. $]$ This model accounted for $82 \%$ (vs. $75 \%$ in model $\mathrm{I}$ ) of the variance in the change in BMI. Adjusting for multiple testing using the permutation test (5000 permutations), however, showed weak evidence that socioeconomic status was associated with change in BMI $(p=0.056)$.
}

None of the hypothesized sample or intervention features (see Moderator Analyses) were associated with BMI at the final assessment (Supplementary Table S4).

\section{Risk of Bias within Studies}

The overall risk of bias judgments revealed that most studies were at low risk of bias $(40 \%)$ or provided insufficient details to determine the quality of the study (35\%). Most studies were judged to be at low risk for selection $(53 \%)$, attrition $(49 \%)$, or other sources $(61 \%)$ of bias. The studies were typically judged to be at high risk for bias due to inadequate allocation concealment $(51 \%)$, performance bias $(59 \%)$, or detection bias $(39 \%)$ because concealing the allocation and blinding of participants or personnel was not possible for many of the studies. Reporting biases (i.e., selective reporting) were typically judged as low (43\%) or unclear $(43 \%)$ risk of bias. The risk of bias score was not associated with the overall BMI at the immediate $(p=0.943)$ or final $(p s=0.343)$ assessment (data not shown). The overall judgment and ratings for the seven sources of bias can be found in Supplementary Figure S1.

\section{Risk of Bias across Studies}

Funnel plots and results of the statistical tests to assess for the possibility of publication bias appear in the online appendix supporting this article, available in the Supplementary Data S2. The graphical and statistical tests revealed asymmetries that might be interpreted as smallstudy effects for BMI at the last follow-up assessment. Trim-and-fill procedures were used to estimate the number of potentially "missing" studies and the pooled ES (using random-effects procedures) if the presumed to be "missing" studies were included. These tests indicated that three studies may have been omitted. The pooled ES estimate was consistent with the original pooled ES for overall BMI, suggesting that including the "missing" studies would not change the overall conclusion $(g=0.17,95 \% \mathrm{CI}=0.04$ 0.30 and estimated $g=0.13,95 \% \mathrm{CI}=0.04-0.23$ ).

\section{Discussion}

The current systematic review and meta-analysis shows that interventions to improve weight status in preschool-aged children are effective in reducing BMI. The overall magnitudes of the ESs are small $(g=0.10$ at the end of treatment and $g=0.17$ at the last follow-up) but suggest that $54 \%$ and $58 \%$ of the children exposed to an intervention had a lower BMI relative to the average for children in the control or comparison group at the immediate and final assessments, respectively. The magnitudes of ESs were likely impacted by our ES adjustments for baseline BMI values in consideration of regression to the mean (i.e., a statistical phenomenon that arises if extreme values observed in a group at baseline are closer to the mean at follow-up). Our findings, however, may be limited to certain settings or populations (e.g., clinical settings, children of low 


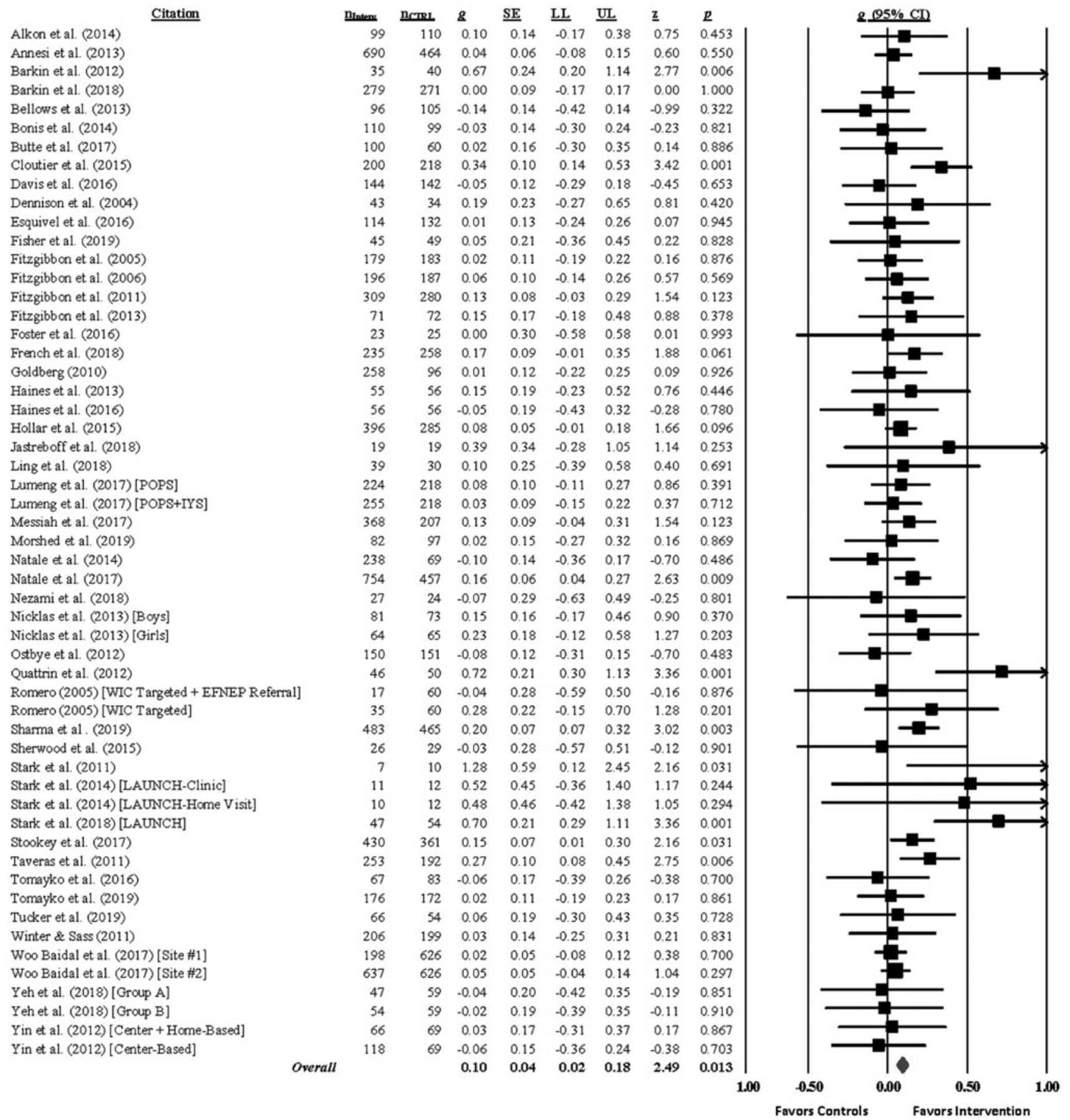

Figure 2. Childhood Obesity Evidence Base Project Forest Plot of the Impact of Child Obesity Interventions on BMI at the Immediate Post-intervention Assessment $(k=55){ }^{* a}$ a Random effects (full information maximum likelihood) model. The size of the square representing the standardized mean difference for each study is proportional to its weight in the analysis and the line running through the square represents the $95 \%$ CI. The diamond represents the overall standardized mean difference and the width of the diamond represents the $95 \%$ CI. Heterogeneity: $I^{2}=20 \%(95 \%$ CI $=0-43) ; \tau^{2}=0.0303 ; Q(54)=67.08, p=0.109$. CI, confidence interval.

socioeconomic status) and future meta-analyses should continue to examine which settings or populations benefit most from these interventions as the scientific literature grows. Nevertheless, our findings indicate that children aged 2-5 years who participate in an obesity intervention gain less BMI over time than children in the control or comparison groups. It is important to note that this outcome is not a prevalence reduction, but it indicates a slowing of obesity in the intervention relative to the comparison condition as measured by lower weight status. The limited heterogeneity observed for overall BMI provides strong clinical evidence supporting the use of obesity prevention interventions for children $2-5$ years of age living in the United States. 


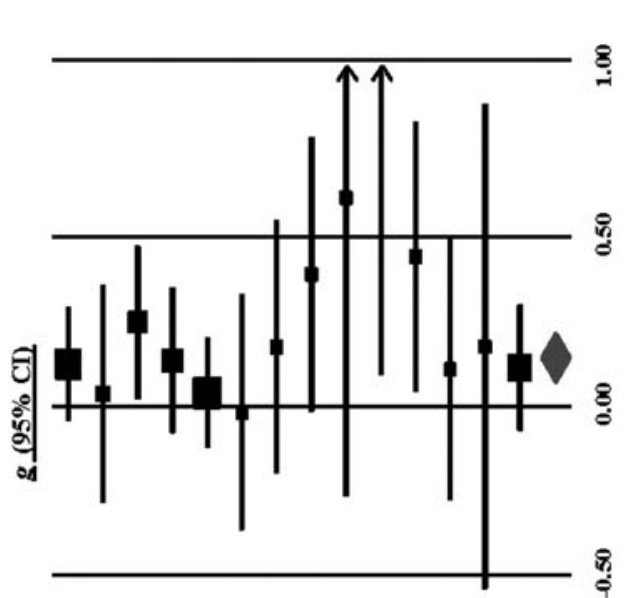

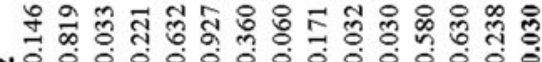

NI

สิ

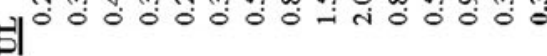

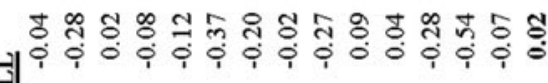

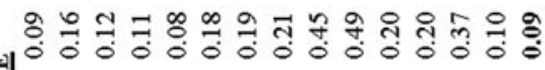
엥

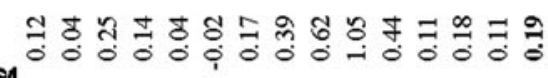

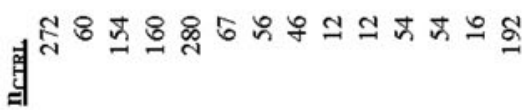

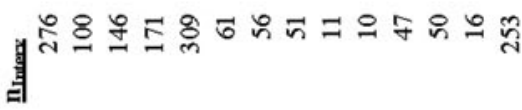

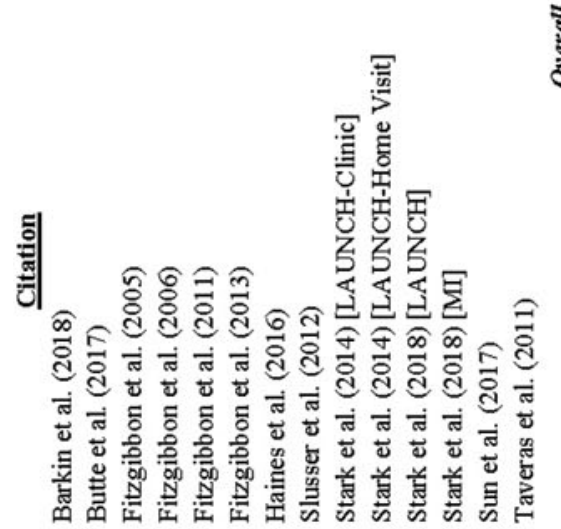

列

This meta-analysis represents the first use of a taxonomy approach to identify components used in early childhood obesity interventions. Taxonomic meta-analysis allows for the discovery of components or patterns of components that are critical for behavioral change approaches, ${ }^{60}$ which were included as moderators in this analysis. Four prior systematic reviews in the area of childhood obesity prevention have used a taxonomical approach to identify intervention components but these reviews focused on infant feeding interventions, ${ }^{61}$ parent-involved childhood weight control interventions for children $0-12$ years of age, ${ }^{62}$ or prevention and management of childhood obesity in children and adolescents aged 1-18 years $^{63}$ or $2-18$ years. ${ }^{64}$ These systematic reviews were limited in scope, reviewing fewer than 24 studies each, and used a pre-existing taxonomy of behavioral change techniques that was not developed specifically to identify intervention components in the area of childhood obesity. ${ }^{65,66}$

The current systematic review and meta-analysis extended prior research by focusing on any childhood obesity intervention targeted toward children aged 2-5 years, used the grounded theory ${ }^{16,17}$ within the SEM framework to identify the specific components of the intervention, ${ }^{19}$ and used meta-regression to identify whether intervention components impacted the efficacy of the findings. The development, refinement, and application of the taxonomy was a collaboration of a multidisciplinary team of experts, and they represent a significant step toward identifying the "active ingredients" of childhood obesity interventions, but ongoing applications will be necessary to optimize reliability, comprehensiveness, and implementation of the taxonomy especially as new studies emerge. Nevertheless, it is noteworthy that the findings from the taxonomic analysis performed in COEB support other taxonomic analyses based on behavior change components expected to affect weight outcomes. ${ }^{65,66}$

Taxonomic meta-regression analyses identified three components that improved the success of the intervention in lowering children's BMI at the end of treatment: Engage caregivers in praise or encouragement for positive healthrelated behavior (e.g., positive affirmation or encouragement to child from caregivers), provide education about the importance of screen time reduction to caregivers, and engage pediatrician or health care providers in delivering all or part of the intervention content. However, when the intervention components were simultaneously entered in a regression model, providing education about the importance of screen time reduction to caregivers was the only significant moderator of BMI change. This model accounted for $89 \%$ of the variance in the change of BMI, suggesting the validity of the taxonomy of intervention components.

Exploratory subgroup analyses revealed a small but significant effect in reducing BMI in interventions in which a screen time component was present $(g=0.23,95 \%$ $\mathrm{CI}=0.10-0.36)$ versus absent $(g=0.05,95 \% \mathrm{CI}=-0.02$ to 0.12 ) relative to the control or comparison group $\left[Q_{\mathrm{B}}\right.$ $(1)=11.24, p=<0.001]$. This is an important finding given that screen time habits in children younger than the age 
of three are associated with increased screen use at 8 years of age, ${ }^{67}$ and the robustness of research showing that screen time exposure is associated with an increased risk of obesity. ${ }^{68}$ This also highlights the critical role of parents in reducing childhood obesity, ${ }^{69}$ and the need to identify specific strategies and provide skills-training to support parents of preschoolers who often find it challenging to limit screen time exposure ${ }^{70}$ These findings are consistent with the Community Preventive Services Task Force, which found strong evidence supporting the efficacy of interventions addressing screen time reduction to promote healthy weight in children aged 13 and younger. ${ }^{71}$ Promoting screen time reduction could have the additional benefit of improving children's physical activity, diet, and sleep behaviors. ${ }^{72}$ Further, as young children five must receive required vaccinations to attend public school, ${ }^{73}$ engaging health care teams (e.g., physician, nurse, medical assistant) in delivery of obesity prevention initiatives seems both feasible and pragmatic given that half $(53 \%)$ of the studies that promoted screen time reduction recruited children from clinical settings. The moderators highlighting the importance of the clinical setting (recruitment and implementation) may have explained additional between-study variance but were not included in the final multiple meta-regression model due to multicollinearity and overfitting of the model.

The success of childhood obesity prevention interventions to improve weight status was also associated with the characteristics of the samples. First, consistent with our hypothesis, childhood obesity interventions sampling a greater proportion of children meeting criteria for overweight or obesity were more successful in improving weight status. Other reviews corroborate our findings showing that multicomponent obesity interventions can reduce weight status in overweight or obese preschoolers, but this moderator was no longer significant when screen time reduction was included in our exploratory models, suggesting that interventions providing education regarding screen time reduction to caregivers are beneficial for normal weight, overweight, and obese children. This finding is also consistent with the Community Preventive Services Task Force, which showed that interventions to reduce recreational sedentary screen time were effective regardless of weight status. ${ }^{71,72}$

Second, as hypothesized, childhood obesity interventions sampling children of lower socioeconomic status were less successful in reducing BMI. This is an important and concerning finding given that low socioeconomic status is a critical risk factor for childhood obesity, and early prevention efforts are critical to prevent obesity in children of lower socioeconomic status. Exploratory multiple meta-regression analyses, however, showed weakened evidence that low socioeconomic status is associated with intervention efficacy when adjusting for multiple moderators. These finding suggest that traditional prevention strategies such as reducing screen time may also require content to address upstream (e.g., reducing poverty, increasing access to education) and midstream (e.g., reducing poverty-related stress) risk factors to reduce so- cioeconomic disparities in obesity, ${ }^{20}$ and we encourage researchers to conduct future studies evaluating novel intervention strategies to address socioeconomic adversity.

Finally, other hypothesized characteristics of the interventions (implementation in a school-based setting, targeting nutrition and physical activity, and involving caregivers) did not emerge as significant moderators of the intervention efficacy. Some reviews have found moderately strong evidence supporting the efficacy of interventions implemented in a schoolbased setting, diet combined with physical activity interventions, or those involving caregivers, but these have largely been found in meta-analyses sampling of children from a wider age range (e.g., 2-18 years of age), multiple geographic locations, or limited to specific intervention settings (e.g., schools). ${ }^{2-12,74}$ Such distinctions highlight the utility of a taxonomic metaanalytic approach to more precisely identify the intended recipients, context, and channels of delivery to improve the effectiveness of early childhood obesity interventions.

\section{Limitations}

Several limitations should be considered when interpreting the findings from this meta-analysis. First, as with any meta-analysis, identifying relevant studies is limited to the reports included in the electronic bibliographic database, our primary source for retrieving relevant reports. To reduce the possibility of omitting potentially relevant research, we conducted a comprehensive search of multiple bibliographic databases, cross-checked our findings with registries of federal grant funding and clinical trials, and consulted with experts in the field. It remains unclear, however, whether only the most rigorous interventions contributed most of the data, or whether all available data from community-based obesity prevention efforts in this age range were captured by using our search methods.

Second, we intended to include all studies evaluating an intervention to prevent or reduce childhood obesity in this age group but limitations in study design (e.g., no control or comparison condition), sample (e.g., children with a mean age $<2$ or $>5$ years of age), or outcomes (i.e., no objective measure of BMI reported) impacted the number of reports available for inclusion. Also, by insisting that BMI be an inclusion criterion, it may be that the sample of studies was not representative of the many communitybased intervention programs that focus on policy, system, and environmental changes to influence healthy behavior change. Third, we included studies that provided transformations of BMI (i.e., BMI z-score, BMI percentile) that are known to be weakly associated with other measures of body fat in children with severe obesity and may have obscured changes in BMI in samples with a greater proportion of children in the 95 th percentile or greater. ${ }^{75}$

Finally, our moderator tests were limited to the intervention descriptions provided in the studies and supplemental reports. Intervention content is often not described in full detail due to space limitations in journals, and intervention fidelity was only addressed in one-third of the included studies. Further, the length of exposure to specific 
intervention components is typically not provided. Therefore, we may have been limited to find effects for some of the intervention components or strategies because those components were not well represented in the interventions or reported fully in the database of included studies (i.e., insufficient statistical power and restriction of range). Methods described in implementation science evaluation schemas can provide guidance for accomplishing this goal. ${ }^{76}$ Replication of this meta-analytic approach by using another age range or targeted outcomes (e.g., improving healthy eating; increasing physical activity; reducing screen time across age groups) will be necessary to demonstrate the robustness of using this taxonomic approach.

\section{Conclusion}

This is the first meta-analysis to use a taxonomic approach to identify components used in early childhood obesity interventions. Findings show that early childhood obesity interventions efforts may be successful in reducing the rate of increase in BMI over time, and that these changes may persist for up to 3 years after the intervention. Specific intervention components emerged as promising strategies: Training caregivers in healthy behavior change strategies (i.e., reducing screen time), engaging health care providers directly in the delivery of obesity prevention efforts, and using health care settings were effective. Importantly, educating caregivers about the importance of screen time reduction appears to be the most promising intervention component identified in the current metaanalysis, but future research is needed to determine the benefits of delivering this component for all populations (e.g., children of lower socioeconomic status). In sum, we found that using a taxonomic meta-analytic approach does elucidate promising intervention components and strategies that may accelerate efforts to reduce rates of childhood obesity. The taxonomic approach to meta-analysis of study effects allows evaluation of these questions and adds to the information provided by traditional meta-analysis.

\section{Acknowledgments}

This work is a collaborative effort between Mission Measurement and the National Collaborative on Childhood Obesity Research (NCCOR). It was funded by Office of Behavioral and Social Science Research, National Institutes of Health, and guided by members of NCCOR. The four organizations represented in NCCOR are: The Centers for Disease Control and Prevention (CDC); National Institutes of Health (NIH); Robert Wood Johnson Foundation (RWJF); and the United States Department of Agriculture (USDA). Members of the NCCOR Childhood Obesity Evidence Base (COEB) project Working Group include Sonia Arteaga, Christine Hunter, Young Jo, Laura Kettel Khan, and Deborah Young-Hyman. Members of the COEB External Expert Panel (EEP) include Leann Birch, John Cawley, Jamie Chriqui, Angie Cradock, Christina Economos, Debra Haire-Joshu,
Shiriki Kumanyika, Bruce Lee, Lorrene Ritchie, Thomas Robinson, and Marlene Schwartz. We are grateful to the Systematic Review and Meta-Analysis Research Methods Team, especially Melissa M. Feulner, Brittany L. Balletto, and Julie DeCosta for their efforts on this project. This Childhood Obesity Supplement is dedicated to Dr. Leann L. Birch, who passed May 26, 2019, for a life's work devoted to childhood nutrition and health.

\section{Funding Information}

The research reported in this article was supported by the Office of Behavioral and Social Sciences Research of the $\mathrm{NIH}$ under contract number GS-00F-0007M. The findings and conclusions in this article are those of the authors and do not necessarily represent the official position of the NIH or the Centers for Disease Control and Prevention.

\section{Author Disclosure Statement}

No competing financial interests exist.

\section{Supplementary Material}

Supplementary Table S1

Supplementary Table S2

Supplementary Table S3

Supplementary Table S4

Supplementary Figure S1

Supplementary Data S1

Supplementary Data S2

\section{References}

1. Skinner AC, Ravanbakht SN, Skelton JA, et al. Prevalence of obesity and severe obesity in US children, 1999-2016. Pediatrics 2018;141:e20173459.

2. Berge JM, Everts JC. Family-based interventions targeting childhood obesity: A meta-analysis. Child Obes 2011;7:110-121.

3. Bluford DA, Sherry B, Scanlon KS. Interventions to prevent or treat obesity in preschool children: A review of evaluated programs. Obesity 2007;15:1357-1372.

4. Brown T, Moore TH, Hooper L, et al. Interventions for preventing obesity in children. Cochrane Database Syst Rev 2019;7:CD001871.

5. Foster BA, Farragher J, Parker P, Sosa ET. Treatment interventions for early childhood obesity: A systematic review. Acad Pediatr 2015;15:353-361.

6. Katz DL, O'Connell M, Njike VY, et al. Strategies for the prevention and control of obesity in the school setting: Systematic review and meta-analysis. Int J Obes 2008;32:1780-1989.

7. Monasta L, Batty GD, Macaluso A, et al. Interventions for the prevention of overweight and obesity in preschool children: A systematic review of randomized controlled trials. Obes Rev 2011;12:e119-e129.

8. Seburg EM, Olson-Bullis BA, Bredeson DM, et al. A review of primary care-based childhood obesity prevention and treatment interventions. Curr Obes Rep 2015;4:191-197.

9. Showell NN, Fawole O, Segal J, et al. A systematic review of home-based childhood obesity prevention studies. Pediatrics 2013;132:e109-e118. 
10. van Hoek E, Feskens EJ, Bouwman LI, Janse AJ. Effective interventions in overweight or obese young children: Systematic review and meta-analysis. Child Obes 2014;10:448-460.

11. Wang Y, Cai $\mathrm{L}, \mathrm{Wu} \mathrm{Y}$, et al. What childhood obesity prevention programmes work? A systematic review and meta-analysis. Obes Rev 2015;16:547-565.

12. Wang X, Zhou G, Zeng J, et al. Effect of educational interventions on health in childhood: A meta-analysis of randomized controlled trials. Public Health 2018;164:134-147.

13. Daniels SR. The use of BMI in the clinical setting. Pediatrics 2009;124(Suppl 1):S35-S41.

14. What Works, Under What Circumstances, and How? U.S. Department of Health and Human Services: Washington, DC, 2016.

15. Ma J, Lewis MA, Smyth JM. Translational behavioral medicine for population and individual health: Gaps, opportunities, and vision for practice-based translational behavior change research. Transl Behav Med 2018;8:753-760.

16. Glaser BG. Conceptualization: On theory and theorizing using grounded theory. Int J Qual Methods 2002;1:23-38.

17. Strauss A, Corbin J. Grounded theory methodology: An overview. In: Denzin NK, Lincoln YS (Eds.), Handbook of Qualitative Research. Sage Publications, Inc.: Thousand Oaks, CA, 1994, pp. 273-285.

18. McLeroy KR, Bibeau D, Steckler A, Glanz K. An ecological perspective on health promotion programs. Health Educ Q 1988; 15:351-377.

19. King H, Magnus M, Hedges LV, et al. Childhood obesity evidence project: Methods for taxonomy development for application in taxonomic meta-analysis. Mission Measurement: Chicago, IL, 2020.

20. Hemmingsson E. Early childhood obesity risk factors: Socioeconomic adversity, family dysfunction, offspring distress, and junk food self-medication. Curr Obes Rep 2018;7:204-209.

21. Sharifi M, Sequist TD, Rifas-Shiman SL, et al. The role of neighborhood characteristics and the built environment in understanding racial/ethnic disparities in childhood obesity. Prev Med 2016;91:103-109.

22. Kral TVE, Moore RH, Chittams J, et al. Identifying behavioral phenotypes for childhood obesity. Appetite 2018;127:87-96.

23. Brown CL, Halvorson EE, Cohen GM, et al. Addressing childhood obesity: Opportunities for prevention. Pediatr Clin North Am 2015;62:1241-1261.

24. Cooper H, Hedges LV, Valentine JC. The Handbook of Research Synthesis and Meta-Analysis. Russell Sage Foundation: New York, NY, 2009.

25. Lipsey MW, Wilson DB. Practical Meta-Analysis. Sage: Thousand Oaks, CA, 2001

26. Moher D, Liberati A, Tetzlaff J, Altman DG. Preferred reporting items for systematic reviews and meta-analyses: The PRISMA statement. Ann Intern Med 2009;151:264-269, w264.

27. Center for Reviews and Dissemination. Systematic reviews: CRD's guidance for undertaking reviews in health care. 2008. Available at https://www.york.ac.uk/media/crd/Systematic_Reviews.pdf Last accessed November 16, 2019.

28. EndNote X8.2 for Windows and Macintosh [computer program]. Philadelphia, PA: Thomson Reuters, 2018.

29. Higgins JPT, Altman DG, Gøtzsche PC, et al. The Cochrane Collaboration's tool for assessing risk of bias in randomised trials. BMJ 2011;343:d5928.

30. Becker BJ. Synthesizing standardized mean-change measures. Br J Math Stat Psychol 1988;41:257-278.
31. Morris SB, DeShon RP. Combining effect size estimates in metaanalysis with repeated measures and independent-groups designs. Psychol Methods 2002;7:105-125.

32. Sanchez-Meca J, Marin-Martinez F, Chacon-Moscoso S. Effectsize indices for dichotomized outcomes in meta-analysis. Psychol Methods 2003;8:448-467.

33. Hedges LV, Olkin I. Statistical Methods for Meta-Analysis. Academic Press: New York, 1985

34. Johnson BT, Eagly AH. Meta-analysis of research in social and personality psychology. In: Reis HT, Judd CM (eds), Handbook of Research Methods in Social and Personality Psychology, 2nd ed. Cambridge University Press: London, 2014, pp. 675-707.

35. Veroniki AA, Jackson D, Viechtbauer W, et al. Methods to estimate the between-study variance and its uncertainty in metaanalysis. Res Synth Methods 2016;7:55-79.

36. Higgins JPT, Thompson SG. Quantifying heterogeneity in a meta-analysis. Stat Med 2002;21:1539-1558.

37. Huedo-Medina TB, Sanchez-Meca J, Marin-Martinez F, Botella J. Assessing heterogeneity in meta-analysis: Q statistic or I2 index? Psychol Methods 2006;11:193-206.

38. Higgins JPT, Thompson SG, Deeks JJ, Altman DG. Measuring inconsistency in meta-analyses. Br Med J 2003;327:557-560.

39. Stata/SE [computer program]. Version 15.1 for Windows. StataCorp LP: College Station, TX, 2018.

40. Wilson D B. Meta-analysis macros for SAS, SPSS, and Stata. 2005. http://mason.gmu.edu/ dwilsonb/ma.html Last accessed August 22, 2020.

41. Sutton AJ. Publicaion bias. In: Cooper H, Hedges LV, Valentine JC (eds), The Handbook of Research Synthesis and Meta-Analysis. Russel Sage Foundation: New York, NY, 2009, pp. 435-452.

42. Sterne JA, Egger M. Funnel plots for detecting bias in meta-analysis: Guidelines on choice of axis. J Clin Epidemiol 2001;54:1046-1055.

43. Egger M, Davey Smith G, Schneider M, Minder C. Bias in metaanalysis detected by a simple, graphical test. Br Med J 1997;315: 629-634.

44. Begg CB, Mazumdar M. Operating characteristics of a rank correlation test for publication bias. Biometrics 1994;50:1088-1101.

45. Duval S, Tweedie R. Trim and fill: A simple funnel-plot-based method of testing and adjusting for publication bias in metaanalysis. Biometrics 2000;56:455-463.

46. Harbord RM, Higgins JPT. Meta-regression in Stata. Stata $J$ 2008;8:493-519.

47. Fitzgibbon ML. Hip Hop to Health: An evidence-based obesity prevention curriculum for early childhood classrooms. Institute for Health Research \& Policy: University of Illinois of Chicago: Chicago, 2016, p. 71.

48. Lumeng JC, Miller AL, Horodynski MA, et al. Improving selfregulation for obesity prevention in head start: A randomized controlled trial. Pediatrics 2017;139. [Epub ahead of print; DOI: 10.1542/peds.2016-2047.]

49. Romero A. Prevention of childhood obesity among low-income preschool-aged children. MS. Utah State University: Ann Arbor, 2005.

50. Stark LJ, Clifford LM, Towner EK, et al. A pilot randomized controlled trial of a behavioral family-based intervention with and without home visits to decrease obesity in preschoolers. $J$ Pediatr Psychol 2014;39:1001-1012.

51. Stark LJ, Spear Filigno S, Bolling C, et al. Clinic and home-based behavioral intervention for obesity in preschoolers: A randomized trial. J Pediatr 2018;192:115-121.

52. Woo Baidal JA, Nelson CC, Perkins M, et al. Childhood obesity prevention in the women, infants, and children program: Outcomes of the MA-CORD study. Obesity 2017;25:1167-1174. 
53. Yeh Y, Hartlieb KB, Danford C, Jen KC. Effectiveness of nutrition intervention in a selected group of overweight and obese African-American preschoolers. J Racial Ethn Health Disparities 2018;5:553-561.

54. Yin Z, Parra-Medina D, Cordova A, et al. Míranos! Look at us, we are healthy! An environmental approach to early childhood obesity prevention. Child Obes 2012;8:429-439.

55. Cloutier MM, Wiley J, Huedo-Medina T, et al. Outcomes from a pediatric primary care weight management program: Steps to growing up healthy. J Pediatr 2015;167:372.e1-377.e1.

56. Goldberg P. A comparison of BMI percentiles and predictor variables for two Head Start childhood obesity prevention programs. Dissertation. School of Nursing, George Mason University: Fairfax, VA, 2010

57. Ling J, Robbins LB, Zhang N, et al. Using Facebook in a healthy lifestyle intervention: Feasibility and preliminary efficacy. West $J$ Nurs Res 2018;40:1818-1842.

58. Sharma SV, Vandewater E, Chuang RJ, et al. Impact of the coordinated approach to child health early childhood program for obesity prevention among preschool children: The Texas Childhood Obesity Research Demonstration study. Child Obes 2019;15:1-13.

59. Slusser W, Frankel F, Robison K, et al. Pediatric overweight prevention through a parent training program for 2-4 year old Latino children. Child Obes 2012;8:60-70.

60. Hedges LV, Saul JA, Cyr C, et al. Childhood Obesity Evidence Base Project: A rationale for taxonomic versus conventional metaanalysis. Childhood Obes 2020;16:S2-1-S2-6.

61. Matvienko-Sikar K, Toomey E, Delaney L, et al. Behaviour change techniques and theory use in healthcare professionaldelivered infant feeding interventions to prevent childhood obesity: A systematic review. Health Psychol Rev 2019;16:1-18.

62. van der Kruk JJ, Kortekaas F, Lucas C, Jager-Wittenaar H. Obesity: A systematic review on parental involvement in longterm European childhood weight control interventions with a nutritional focus. Obes Rev 2013;14:745-760.

63. Golley RK, Hendrie GA, Slater A, Corsini N. Interventions that involve parents to improve children's weight-related nutrition intake and activity patterns - what nutrition and activity targets and behaviour change techniques are associated with intervention effectiveness? Obes Rev 2011;12:114-130.

64. Martin J, Chater A, Lorencatto F. Effective behaviour change techniques in the prevention and management of childhood obesity. Int J Obes 2013;1137:1287-1294.

65. Michie S, Carey RN, Johnston M, et al. From theory-inspired to theory-based interventions: A protocol for developing and testing a methodology for linking behaviour change techniques to theoretical mechanisms of action. Ann Behav Med 2017;52:501-512.

66. Michie S, Ashford S, Sniehotta FF, et al. A refined taxonomy of behaviour change techniques to help people change their physical activity and healthy eating behaviours: The CALO-RE taxonomy. Psychol Health 2011;26:1479-1498.

67. Trinh MH, Sundaram R, Robinson SL, et al. Association of trajectory and covariates of children's screen media time. JAMA Pediatr 2019;174:71-78.

68. Robinson TN, Banda JA, Hale L, et al. Screen media exposure and obesity in children and adolescents. Pediatrics 2017;140(Suppl 2): S97-S101.

69. Lindsay AC, Sussner KM, Kim J, Gortmaker S. The role of parents in preventing childhood obesity. Future Child 2006;16: 169-186.

70. Hesketh KD, Hinkley T, Campbell KJ. Children's physical activity and screen time: Qualitative comparison of views of parents of infants and preschool children. Int J Behav Nutr Phys Act 2012;9:152.
71. Community Prevention Services Task Force. Obesity prevention and control: Behavioral interventions that aim to reduce recreational sedentary screen time among children. 2015. Available at https:// www.thecommunityguide.org/sites/default/files/assets/ObesityBehavioral-Screentime.pdf Last accessed November 26, 2019.

72. Ramsey Buchanan L, Rooks-Peck CR, Finnie RKC, et al. Reducing recreational sedentary screen time: A community guide systematic review. Am J Prev Med 2016;50:402-415.

73. Barraza L, Schmit C, Hoss A. The latest in vaccine policies: Selected issues in school vaccinations, healthcare worker vaccinations, and pharmacist vaccination authority laws. J Law Med Ethics 2017;45(Suppl 1):16-19.

74. Birch LL, Ventura AK. Preventing childhood obesity: What works? Int $J$ Eat Disord 2009;42:284-289.

75. Freedman DS, Butte NF, Taveras EM, et al. BMI z-scores are a poor indicator of adiposity among 2- to 19-year-olds with very high BMIs, NHANES 1999-2000 to 2013-2014. Obesity (Silver Spring) 2017;25:739-746.

76. Leeman J, Birken SA, Powell BJ, et al. Beyond "implementation strategies": Classifying the full range of strategies used in implementation science and practice. Implement Sci 2017;12:125.

77. Alkon A, Crowley AA, Neelon SE, et al. Nutrition and physical activity randomized control trial in child care centers improves knowledge, policies, and children's body mass index. $B M C$ Public Health 2014;14. [Epub ahead of print; DOI: 10.1186/ 1471-2458-14-215.]

78. Ammerman AS, Ward DS, Benjamin SE, et al. An intervention to promote healthy weight: Nutrition and Physical Activity SelfAssessment for Child Care (NAP SACC): Theory and design. Prev Chronic Dis 2007;4:1-12.

79. Kotch JB. Child Care Wellness Study on Nutrition and Physical Activity (CCWS). 2013. Available at https://clinicaltrials.gov/ct2/ show/record/NCT01921842?term $=$ NCT01921842\&rank=1 Last accessed October 15, 2019.

80. Annesi JJ, Smith AE, Tennant GA. Effects of a cognitive-behaviorally based physical activity treatment for 4- and 5-year-old children attending US preschools. Int J Behav Med 2013;20:562-566.

81. Annesi JJ, Smith AE, Tennant GA. Effects of the Start for Life treatment on physical activity in primarily African American preschool children of ages 3-5 years. Psychol Health Med 2013; 18:300-309.

82. Annesi JJ, Smith AE, Tennant GA. Reducing high BMI in African American preschoolers: Effects of a behavior-based physical activity intervention on caloric expenditure. South Med J 2013; 106:456-459.

83. Annesi JJ, Smith AE, Tennant GA. Cognitive-behavioural physical activity treatment in African-American pre-schoolers: Effects of age, sex, and BMI. $J$ Paediatr Child Health 2013;49:E128-E132.

84. Barkin SL, Gesell SB, Po'e EK, et al. Culturally tailored, familycentered, behavioral obesity intervention for Latino-American preschool-aged children. Pediatrics 2012;130:445-456.

85. Ruiz RM, Gesell SB, Buchowski MS, et al. The relationship between Hispanic parents and their preschool-aged children's physical activity. Pediatrics 2011;127:888-895

86. Barkin SL. ST of TN-Salud Con La Familia: Prevent Onset of Ped Obesity. 2008. Available at https://clinicaltrials.gov/ct2/ show/NCT00808431 Last accessed August 15, 2019.

87. Barkin SL, Heerman WJ, Sommer EC, et al. Effect of a behavioral intervention for underserved preschool-age children on change in body mass index: A randomized clinical trial. JAMA 2018;320:450-460.

88. Po'e EK, Heerman WJ, Mistry RS, Barkin SL. Growing Right Onto Wellness (GROW): A family-centered, community-based 
obesity prevention randomized controlled trial for preschool child-parent pairs. Contemp Clin Trials 2013;36:436-449.

89. White RO, Thompson JR, Rothman RL, et al. A health literate approach to the prevention of childhood overweight and obesity. Patient Educ Couns 2013;93:612-618.

90. Cui Z, Truesdale KP, Robinson TN, et al. Recruitment strategies for predominantly low-income, multi-racial/ethnic children and parents to 3-year community-based intervention trials: Childhood Obesity Prevention and Treatment Research (COPTR) Consortium. Trials 2019;20:296.

91. Ruiz RM, Sommer EC, Tracy D, et al. Novel patterns of physical activity in a large sample of preschool-aged children. BMC Public Health 2018;18:242.

92. LeCroy MN, Truesdale KP, Matheson DM, et al. Snacking characteristics and patterns and their associations with diet quality and BMI in the Childhood Obesity Prevention and Treatment Research Consortium. Public Health Nutr 2019;21:1-11.

93. Heerman WJ, Taylor JL, Wallston KA, Barkin SL. Parenting selfefficacy, parent depression, and healthy childhood behaviors in a low-income minority population: A cross-sectional analysis. Matern Child Health J 2017;21:1156-1165.

94. Barkin SL, Lamichhane AP, Banda JA, et al. Parent's physical activity associated with preschooler activity in underserved populations. Am J Prev Med 2017;52:424-432.

95. Heerman WJ, Mitchell SJ, Thompson J, et al. Parental perception of built environment characteristics and built environment use among Latino families: A cross-sectional study. PLoS One 2016;16:1180.

96. LeCroy MN, Truesdale KP, Matheson D, et al. Snacking characteristics and patterns in the childhood obesity prevention and treatment research consortium. Public Health Nutr 2019; 22:3189-3199.

97. Barkin SL. Growing Right Onto Wellness (GROW): Changing early childhood body mass index (BMI) trajectories (GROW). 2011. Available at https://clinicaltrials.gov/ct2/show/record/ NCT01316653?view=record Last accessed October 15, 2019.

98. Bellows LL, Davies PL, Anderson J, Kennedy C. Effectiveness of a physical activity intervention for Head Start preschoolers: A randomized intervention study. Am J Occup Ther 2013;67:28-36.

99. Bellows LL, Anderson J. The food friends get movin' with mighty moves: A physical activity program for preschoolers. $J$ Nutr Educ Behav 2013;45:473-475.

100. Bellows LL, Anderson J, Gould SM, Auld G. Formative research and strategic development of a physical activity component to a social marketing campaign for obesity prevention in preschoolers. $J$ Community Health 2008;33:169-178.

101. Bellows LL, Silvernail S, Caldwell L, et al. Parental perception on the efficacy of a physical activity program for preschoolers. $J$ Community Health 2011;36:231-237.

102. Bellows LL, Gavin WJ, Johnson SL, et al. Obesity prevention study: A physical activity program improved gross motor skills in preschool children. Am J Occup Ther 2015;69(Suppl 1).

103. Bellows LL. Development and evaluation of Food Friends Get Movin' with Mighty Moves ${ }^{\mathrm{TM}}$, a physical activity program to prevent obesity in low-income preschoolers. PhD. Food Science and Human Nutrition, Colorado State University: Ann Arbor, 2007.

104. Bonis M, Loftin M, Ward D, et al. Improving physical activity in daycare interventions. Child Obes 2014;10:334-341.

105. Ward DS, Benjamin SE, Ammerman AS, et al. Nutrition and physical activity in child care: Results from an environmental intervention. Am J Prev Med 2008;35:352-356.

106. Butte NF, Hoelscher DM, Barlow SE, et al. Efficacy of a community- versus primary care-centered program for childhood obesity: TX CORD RCT. Obesity 2017;25:1584-1593.
107. Hoelscher DM, Butte NF, Barlow S, et al. Incorporating primary and secondary prevention approaches to address childhood obesity prevention and treatment in a low-income, ethnically diverse population: Study design and demographic data from the Texas Childhood Obesity Research Demonstration (TX CORD) study. Child Obes 2015;11:71-91.

108. Wilson TA, Liu Y, Adolph AL, et al. Behavior modification of diet and parent feeding practices in a community- vs primary care-centered intervention for childhood obesity. J Nutr Educ Behav 2019;51:150-161.

109. Oluyomi AO, Byars A, Byrd-Williams C, et al. The utility of Geographical Information Systems (GIS) in systems-oriented obesity intervention projects: The selection of comparable study sites for a quasi-experimental intervention design-TX CORD. Child Obes 2015;11:1-3.

110. Hoelscher DM. Texas Childhood Obesity Research Demonstration (TX CORD) Project. 2016. Available at https:// clinicaltrials.gov/ct2/show/NCT02724943

111. Barlow SE, Butte NF, Hoelscher DM, et al. Strategies to recruit a diverse low-income population to child weight management programs from primary care practices. Prev Chronic Dis 2017;14:e138.

112. Gorin AA, Wiley J, Ohannessian CM, et al. Steps to growing up healthy: A pediatric primary care based obesity prevention program for young children. BMC Public Health 2014;14:72.

113. Trapp CM, Burke G, Gorin AA, et al. The relationship between dietary patterns, body mass index percentile, and household food security in young urban children. Child Obes 2015;11:148-155.

114. Cloutier MM. The added value of telephone follow up and home visits in helping children to grow up healthy. 2013. Available at https:/clinicaltrials.gov/ct2/show/record/NCT01973153?term= NCT01973153\&rank=1 Last accessed October 15, 2019.

115. Davis SM, Myers OB, Cruz TH, et al. CHILE: Outcomes of a group randomized controlled trial of an intervention to prevent obesity in preschool Hispanic and American Indian children. Prev Med 2016;89:162-168.

116. Davis SM. Child Health Initiative for Lifelong Eating and Exercise (CHILE). 2007. Available at https://clinicaltrials.gov/ct2/ show/NCT00428805 Last accessed June 17, 2019.

117. Sussman AL, Davis S. Integrating formative assessment and participatory research: Building healthier communities in the CHILE Project. Am J Health Ed 2010;41:244-249.

118. Cruz TH, Davis SM, FitzGerald CA, et al. Engagement, recruitment, and retention in a trans-community, randomized controlled trial for the prevention of obesity in rural American Indian and Hispanic children. J Prim Prev 2014;35:135-149.

119. Trappmann JL, Jimenez EY, Keane PC, et al. Cross-sectional relationships between household food insecurity and child BMI, feeding behaviors, and public assistance utilization among head start children from predominantly Hispanic and American Indian communities in the CHILE study. J Hunger Environ Nutr 2015;10:439-455.

120. Cruz TH, Davis SM, Myers OB, et al. Effects of an obesity prevention intervention on physical activity among preschool children. Health Promot Pract 2016;17:693-701.

121. Morshed AB, Davis SM, Keane PC, et al. The impact of the CHILE intervention on the food served in head start centers in rural New Mexico. J Sch Health 2016;86:414-423.

122. Morshed AB, Davis SM, Greig EA, et al. Effect of WIC food package changes on dietary intake of preschool children in New Mexico. Health Behav Policy Rev 2015;2:3-12.

123. Davis SM, Sanders SG, FitzGerald CA, et al. CHILE: An evidence-based preschool intervention for obesity prevention in Head Start. J Sch Health 2013;83:223-229. 
124. Dennison BA, Russo TJ, Burdick PA, Jenkins PL. An intervention to reduce television viewing by preschool children. Arch Pediatr Adolesc Med 2004;158:170-176.

125. Dennison BA. Brocodile the crocodile: Obesity prevention in day care. National Institutes of Health: Cooperstown, NY, 1999.

126. Dennison BA, Faith MS. Prevention of childhood obesity in childcare settings. In: Jelalian E, Steele RG (eds), Handbook of Childhood and Adolescent Obesity. Springer Science + Business Media: New York, NY, 2008, pp. 313-330.

127. Esquivel MK, Nigg CR, Fialkowski MK, et al. Head start wellness policy intervention in Hawaii: A project of the children's healthy living program. Child Obes 2016;12:26-32.

128. Wilken LR, Novotny R, Fialkowski MK, et al. Children's Healthy Living (CHL) Program for remote underserved minority populations in the Pacific region: Rationale and design of a community randomized trial to prevent early childhood obesity. BMC Public Health 2013;13:944.

129. Novotny R, Fialkowski MK, Areta AA, et al. University of Hawai'i Cancer Center connection: The Pacific Way to Child Wellness: The Children's Healthy Living Program for Remote Underserved Minority Populations of the Pacific Region (CHL). Hawaii J Med Public Health 2013;72:406-408.

130. Esquivel MK, Nigg CR, Fialkowski MK, et al. Influence of teachers' personal health behaviors on operationalizing obesity prevention policy in head start preschools: A project of the Children's Healthy Living Program (CHL). J Nutr Educ Behav 2016;48:318-325.

131. Novotny R. Children's Healthy Living Program (CHL). 2013. Available at https://clinicaltrials.gov/ct2/show/NCT01881373 Last accessed August 15, 2019.

132. Fisher JO, Serrano EL, Foster GD, et al. Efficacy of a food parenting intervention for mothers with low income to reduce preschooler's solid fat and added sugar intakes: A randomized controlled trial. Int J Behav Nutr Phys Act 2019;16:6.

133. Fisher JO. Feeding, fun, and families study. 2018. Available at https:/clinicaltrials.gov/ct2/show/NCT03646201 Last accessed November 4, 2019.

134. Fitzgibbon ML, Stolley MR, Schiffer L, et al. Two-year followup results for Hip-Hop to Health Jr.: A randomized controlled trial for overweight prevention in preschool minority children. $J$ Pediatr 2005;146:618-625.

135. Fitzgibbon ML, Stolley MR, Dyer AR, et al. A community-based obesity prevention program for minority children: Rationale and study design for Hip-Hop to Health Jr. Prev Med 2002;34:289-297.

136. Stolley MR, Fitzgibbon ML, Dyer A, et al. Hip-Hop to Health Jr., an obesity prevention program for minority preschool children: Baseline characteristics of participants. Prev Med 2003;36:320-329.

137. Fitzgibbon ML, Stolley MR, Schiffer L, et al. Hip-Hop to Health Jr. for Latino preschool children. Obesity 2006;14:1616-1625.

138. Fitzgibbon ML, Stolley MR, Schiffer LA, et al. Hip-Hop to Health Jr. Obesity Prevention Effectiveness Trial: Postintervention results. Obesity 2011;19:994-1003.

139. Kong A, Buscemi J, Stolley MR, et al. Hip-Hop to Health Jr. randomized effectiveness trial: 1-Year follow-up results. $\mathrm{Am} J$ Prev Med 2016;50:136-144.

140. Buscemi J, Berlin KS, Rybak TM, et al. Health behavior and weight changes among ethnic and racial minority preschoolers and their parents: Associations across 1 year. J Pediatr Psychol 2016;41:777-785.

141. Lown DA, Fitzgibbon ML, Dyer A, et al. Effect of variable energy served on 24-hour energy intake in 16 preschools, Chicago, Illinois, 2007. Prev Chronic Dis 2011;8:A58.

142. Fitzgibbon ML. Prevention of overweight in preschool minority children. National Institutes of Health: Chicago, IL, 1998.
143. Fitzgibbon ML. Preschool based obesity prevention effectiveness trial. 2005. Available at https://clinicaltrials.gov/ct2/show/record/ NCT00241878?term $=$ NCT00241878\&rank=1 Last accessed October 15, 2019.

144. Fitzgibbon ML, Stolley MR, Schiffer L, et al. Family-based hiphop to health: Outcome results. Obesity 2013;21:274-283.

145. Foster BA, Aquino CA, Gil M, et al. A pilot study of parent mentors for early childhood obesity. J Obes 2016;2016:1-13.

146. Foster BA, Aquino C, Gil M, et al. A randomized clinical trial of the effects of parent mentors on early childhood obesity: Study design and baseline data. Contemp Clin Trials 2015;45:164-169.

147. Villanueva GA, Foster BA. Factors associated with successful mentoring of parents addressing childhood obesity: A mixed methods approach. Int J Pediatr 2016;2016. [Epub ahead of print; DOI:10.1155/2016/5769621.]

148. Hollister EB, Foster BA, Dahdouli M, et al. Characterization of the stool microbiome in Hispanic preschool children by weight status and time. Child Obes 2018;14:122-130.

149. Hollister EB. Parent mentors using positive deviance in childhood obesity. 2015. Available at https://clinicaltrials.gov/ct2/show/ record/NCT02373670?term $=$ NCT02373670\&rank $=1$ Last accessed October 15, 2019.

150. French SA, Sherwood NE, Veblen-Mortenson S, et al. Multicomponent obesity prevention intervention in low-income preschoolers: Primary and subgroup analyses of the NET-Works randomized clinical trial, 2012-2017. Am J Public Health 2018; 108:1695-1706.

151. Sherwood NE, French SA, Veblen-Mortenson S, et al. NETWorks: Linking families, communities and primary care to prevent obesity in preschool-age children. Contemp Clin Trials 2013;36:544-554.

152. French SA. NET-Works: Community preschooler obesity prevention (NET-Works). 2012. Available at https://clinicaltrials.gov/ct2/ show/record/NCT01606891?term $=$ NCT01606891\&rank=1 Last accessed October 15, 2019.

153. Allar I, Jones E, Elliott E, et al. The perceived impact of I am moving, I am learning on physical activity and family involvement: A preliminary investigation. Am J Health Behav 2017;41: 683-692.

154. Haines J, McDonald J, O'Brien A, et al. Healthy Habits, Happy Homes: Randomized trial to improve household routines for obesity prevention among preschool-aged children. JAMA Pediatr 2013;167:1072-1079.

155. Taveras EM, McDonald J, O'Brien A, et al. Healthy Habits, Happy Homes: Methods and baseline data of a randomized controlled trial to improve household routines for obesity prevention. Prev Med 2012;55:418-426.

156. Cespedes EM, McDonald J, Haines J, et al. Obesity-related behaviors of US- and non-US-born parents and children in lowincome households. J Dev Behav Pediatr 2013;34:541-548.

157. Taveras EM. Healthy Habits, Happy Homes: An intervention to improve household routines for obesity prevention $(\mathrm{HH})$. 2013. Available at https://clinicaltrials.gov/ct2/show/record/NCT01565161?term= NCT01565161\&rank=1 Last accessed October 15, 2019.

158. Haines J, Rifas-Shiman SL, Gross D, et al. Randomized trial of a prevention intervention that embeds weight-related messages within a general parenting program. Obesity 2016;24: 191-199.

159. Haines J. Parents and tots together: A family-based obesity prevention intervention. 2014. Available at https://clinicaltrialsgov/ show/nct02222766; https://www.cochranelibrary.com/central/ doi/10.1002/central/CN-01583521/full Last accessed July 11, 2019 . 
160. Haines J, Mayorga AM, McDonald J, et al. Embedding weightrelated messages within a general parenting programme: Development and feasibility evaluation of Parents and Tots Together. Early Child Dev Care 2012;182:951-965.

161. Hawkins KR, Apolzan JW, Staiano AE, et al. Efficacy of a homebased parent training-focused weight management intervention for preschool children: The DRIVE randomized controlled pilot trial. J Nutr Educ Behav 2019;51:740-748.

162. Shanley JR. Development of the DRIVE curriculum to address childhood obesity risk factors (clinical trial). 2014. Available at https://clinicaltrials.gov/ct2/show/NCT02160847 Last accessed October 10, 2019.

163. Martin C, Apolzan J, Hawkins K, et al. Efficacy of a home-based weight management intervention for 2-6 year old children and their parents: Results of a randomized controlled pilot trial. Obes Facts 2017;10(Suppl 1):233-234.

164. Hollar D, Heitz C, Zhou W. More young children in an obesity prevention intervention in $\mathrm{ms}$ and la head start centers improve/ maintain BMI percentile and waist circumference compared to nonparticipants. Circulation 2015;131. [Epub ahead of print; DOI: 10.1161/circ.131.suppl_1.mp30.]

165. Jastreboff AM, Chaplin TM, Finnie S, et al. Preventing childhood obesity through a mindfulness-based parent stress intervention: A randomized pilot study. J Pediatr 2018;202:136-142.

166. Sinha R. Family based mindfulness intervention. 2013. Available at https://clinicaltrialsgov/show/nct01974102 Last accessed July 11, 2019.

167. Miller AL, Horodynski MA, Herb HE, et al. Enhancing self-regulation as a strategy for obesity prevention in Head Start preschoolers: The growing healthy study. BMC Public Health 2012;12:1040.

168. Lumeng J, Miller A, Brophy-Herb H, et al. Enhancing selfregulation as a strategy for obesity prevention in Head Start preschoolers. J Nutr Educ Behav 2013;46(4 Suppl):S86.

169. Lumeng JC. Enhancing self regulation as a strategy for obesity prevention in head start preschoolers. 2011. Available at https://clinicaltrials.gov/ct2/show/record/NCT01398358?term= NCT01398358\&rank=1 Last accessed October 15, 2019.

170. Lumeng JC, Miller A, Brophy-Herb H, et al. Enhancing self regulation as a strategy for obesity prevention in head start preschoolers. J Nutr Educ Behav 2015;46(4 Suppl):S195.

171. Messiah SE, Lebron C, Moise R, et al. Healthy caregivers-healthy children (HC2) phase 2: Integrating culturally sensitive childhood obesity prevention strategies into childcare center policies. Contemp Clin Trials 2017;53:60-67.

172. Messiah SE, Natale RA. Healthy Caregivers, Healthy Children (HC2): A childcare center-based obesity prevention program. Presented at the 2017 QRIS National Meeting, Dallas, TX, June 28, 2017.

173. Natale RA. Healthy Caregivers-Healthy Children (HC2) phase II. 2016. Available at https:/clinicaltrials.gov/ct2/show/NCT02697565 Last accessed July 29, 2019

174. Natale RA, Messiah SE, Chang C, et al. Healthy caregivershealthy children: A primary prevention program with preschool children. Cardiology 2016;134(Suppl 1):S71.

175. Natale RA, Messiah SE, Englebert N, et al. Healthy caregivershealthy children: A train-the-trainer (TTT) implemented childcare center-based obesity prevention program. J Nutr Educ Behav 2016;48:S126.

176. Natale RA, Messiah SE. Healthy Caregivers Healthy Children Phase 2 (HC2): Two year follow-up results. J Nutr Educ Behav 2018;50:S117.

177. Morshed AB, Tabak RG, Schwarz CD, Haire-Joshu D. The impact of a healthy weight intervention embedded in a home- visiting program on children's weight and mothers' feeding practices. J Nutr Educ Behav 2019;51:237-244.

178. Tabak RG, Morshed AB, Schwarz CD, Haire-Joshu D. Impact of a healthy weight intervention embedded within a national home visiting program on the home food environment. Front Public Health 2018;6. [Epub ahead of print; DOI: 10.3389/ fpubh.2018.00178.]

179. Haire-Joshu D. Healthy eating and active living taught at home. 2012. Available at https://clinicaltrials.gov/ct2/show/NCT01567033 Last accessed August 22, 2019.

180. Haire-Joshu D, Schwarz CD, Steger-May K, et al. A randomized trial of weight change in a national home visiting program. $\mathrm{Am} \mathrm{J}$ Prev Med 2018;54:341-351.

181. Natale RA, Lopez-Mitnik G, Uhlhorn SB, et al. Effect of a child care center-based obesity prevention program on body mass index and nutrition practices among preschool-aged children. Health Promot Pract 2014;15:695-705.

182. Cespedes EM, Horan CM, Gillman MW, et al. Participant characteristics and intervention processes associated with reductions in television viewing in the High Five for Kids study. Prev Med 2014;62:64-70.

183. Messiah SE, Natale RA, Lopez-Mitnik G, Barth J. Effect of a school-based obesity prevention intervention on body mass index and nutrition in inner-city, multiethnic preschool children. Circulation 2008;117:e199.

184. Natale RA, Messiah SE, Barth J, et al. An obesity prevention program with preschool children: One year follow-up data. Obesity 2008;16:S146.

185. Natale RA, Messiah SE, Asfour LS, et al. Obesity prevention program in childcare centers: Two-year follow-up. Am J Health Promot 2017;31:502-510.

186. Natale RA, Messiah SE, Asfour L, et al. Role modeling as an early childhood obesity prevention strategy: Effect of parents and teachers on preschool children's healthy lifestyle habits. $J$ Dev Behav Pediatr 2014;35:378-387.

187. Natale RA, Scott SH, Messiah SE, et al. Design and methods for evaluating an early childhood obesity prevention program in the childcare center setting. BMC Public Health 2013;13:78.

188. McCollister KE, Tolbert DV, Mishra S, et al. Cost analysis of a childcare center-based intervention to prevent obesity in the preschool years. J Compr Pediatr 2015;6. [Epub ahead of print; DOI: 10.17795/comperped-25845.]

189. Messiah SE, Asfour L, Arheart KL, et al. Relationship between parent demographic characteristics, perinatal and early childhood behaviors, and body mass index among preschool-age children. $J$ Immigr Minor Health 2015;17:414-421.

190. Asfour L, Natale RA, Uhlhorn S, et al. Ethnicity, household food security, and nutrition and activity patterns in families with preschool children. J Nutr Educ Behav 2015;47:498-505.

191. Natale RA, Messiah SE, Asfor L, et al. Healthy CaregiversHealthy Children (HC2): A childcare center-based obesity prevention program. J Nutr Educ Behav 2012;4(Suppl 4):S82.

192. Natale RA, Messiah SE, Asfor L, et al. Healthy CaregiversHealthy Children (HC2): A childcare center based obesity prevention. J Nutr Educ Behav 2013;45(Suppl 4):S86-S87.

193. Natale RA, Uhlhorn SB, Lopez-Mitnik G, et al. Caregiver's country of birth is a significant determinant of accurate perception of preschool-age children's weight. Health Educ Behav 2016;43: 191-200.

194. Natale RA. USDA healthy caregivers/healthy children: A childhood obesity prevention program (HC2). 2012. Available at https://clinicaltrials.gov/ct2/show/NCT01722032 Last accessed July 29, 2019. 
195. Natale RA. Improving the cardiovascular health of preschoolers in a childcare setting. Cardiology 2018;140:1-6.

196. Nezami BT, Ward DS, Lytle LA, et al. A mHealth randomized controlled trial to reduce sugar-sweetened beverage intake in preschool-aged children. Pediatr Obes 2018;13:668-676.

197. Nezami BT, Lytle LA, Tate DF. A randomized trial to reduce sugar-sweetened beverage and juice intake in preschool-aged children: Description of the Smart Moms intervention trial. BMC Public Health 2016;16:837.

198. Nezami BT. Trial of a sugar-sweetened beverage intervention in overweight mothers and their children ages 3-5. 2014. Available at https://clinicaltrials.gov/ct2/show/record/NCT02098902 Last accessed October 15, 2019.

199. Nicklas TA, Nguyen T, Butte NF, Liu Y. The children in action pilot study. Int J Child Health Nutr 2013;2:296-308.

200. Nicklas TA. The Children in Action Feasibility Study (CIA). 2012. Available at https://clinicaltrials.gov/ct2/show/NCT01697124 Last accessed October 8, 2019.

201. Ostbye T, Krause KM, Stroo M, et al. Parent-focused change to prevent obesity in preschoolers: Results from the KAN-DO study. Prev Med 2012;55:188-195.

202. Ostbye T, Zucker NL, Krause KM, et al. Kids and adults now! Defeat Obesity (KAN-DO): Rationale, design and baseline characteristics. Contemp Clin Trials 2011;32:461-469.

203. Ostbye T, Malhotra R, Stroo M, et al. The effect of the home environment on physical activity and dietary intake in preschool children. Int $J$ Obes 2013;37:1314-1321.

204. Ostbye T, Lovelady C. KAN-DO: A family-based intervention to prevent childhood obesity (KAN-DO). 2007. Available at https:// clinicaltrials.gov/ct2/show/record/NCT00563264?term=nct00563264 \&rank=1 Last accessed August 6, 2019.

205. Ostbye T, Fuemmeler B, Malhotra R, et al. The effect of the home environment on directly measured physical activity in young children: Results from Kan-Do. Obes Facts 2012;5:19.

206. Quattrin T, Roemmich JN, Paluch R, et al. Efficacy of familybased weight control program for preschool children in primary care. Pediatrics 2012;130:660-666.

207. Quattrin T, Roemmich JN, Paluch R, et al. Treatment outcomes of overweight children and parents in the medical home. Pediatrics 2014;134:290-297.

208. Quattrin T. Treating pre-school children at risk for overweight in the primary care setting. 2009. Available at https://clinicaltrials.gov/ ct2/show/NCT01029834 Last accessed July 10, 2019.

209. Sharma SV, Chuang RJ, Byrd-Williams C, et al. Using process evaluation for implementation success of preschool-based programs for obesity prevention: The TX Childhood Obesity Research Demonstration Study. J Sch Health 2019;89:382-392.

210. Sherwood NE, JaKa MM, Crain AL, et al. Pediatric primary care based obesity prevention for parents of preschool children: A pilot study. Child Obes 2015;11:674-682.

211. Sherwood NE. Healthy homes/healthy kids preschool. 2010. Available at https://clinicaltrials.gov/ct2/show/NCT01080885 Last accessed July 26, 2019.

212. Slusser W. Detailed description of the nutrition component of a pediatric overweight prevention program: Reply to Anchondo. Child Obes 2013;9:65-66.

213. Stark LJ, Spear S, Boles R, et al. A pilot randomized controlled trial of a clinic and home-based behavioral intervention to decrease obesity in preschoolers. Obesity 2011;19:134-141.

214. Towner EK, Robson SM, Stark LJ. Secondary impact of a behavioral intervention on dietary quality in preschoolers with obesity. Child Health Care 2019;48:75-89.
215. Van Allen J, Kuhl ES, Filigno SS, et al. Changes in parent motivation predicts changes in body mass index z-score (zBMI) and dietary intake among preschoolers enrolled in a family-based obesity intervention. J Pediatr Psychol 2014;39:1028-1037.

216. Stark LJ, Filigno SS, Bolling C, et al. Learning about Activity and Understanding Nutrition for Child Health (LAUNCH): Rationale, design, and implementation of a randomized clinical trial of a family-based pediatric weight management program for preschoolers. Contemp Clin Trials 2017;52:10-19.

217. Stark LJ, Filigno SS, Kichler JC, et al. Maintenance following a randomized trial of a clinic and home-based behavioral intervention of obesity in preschoolers. $J$ Pediatr 2019;213:128-136.

218. Robson SM, Ziegler ML, McCullough MB, et al. Changes in diet quality and home food environment in preschool children following weight management. Int J Behav Nutr Phys Act 2019;16:16.

219. Simon SL, Goetz AR, Meier MF, et al. Sleep duration and bedtime in preschool-age children with obesity: Relation to BMI and diet following a weight management intervention. Pediatr Obes 2019; 14:e12555.

220. Stark LJ. Behavioral treatment for obese preschoolers (LAUNCH). 2012. Available at https://clinicaltrials.gov/ct2/show/record/ NCT01546727?term $=$ NCT01546727\&rank=1 Last accessed October 15, 2019.

221. Stookey JD, Evans J, Chan C, et al. Healthy apple program to support child care centers to alter nutrition and physical activity practices and improve child weight: A cluster randomized trial. BMC Public Health 2017;17:965.

222. Stookey JD. Evaluation of the Healthy Apple Program in San Francisco. 2016. Available at https://clinicaltrials.gov/ct2/show/ NCT02799433 Last accessed August 23, 2019.

223. Sun A, Cheng J, Bui Q, et al. Home-based and technologycentered childhood obesity prevention for chinese mothers with preschool-aged children. J Transcult Nurs 2017;28:616-624.

224. Taveras EM, Gortmaker SL, Hohman KH, et al. Randomized controlled trial to improve primary care to prevent and manage childhood obesity: The High Five for Kids study. Arch Pediatr Adolesc Med 2011;165:714-722.

225. Taveras EM, Hohman KH, Price SN, et al. Correlates of participation in a pediatric primary care-based obesity prevention intervention. Obesity 2011;19:449-452.

226. Sonneville KR, Rifas-Shiman SL, Kleinman KP, et al. Associations of obesogenic behaviors in mothers and obese children participating in a randomized trial. Obesity 2012;20:14491454.

227. Rifas-Shiman SL, Taveras EM, Gortmaker SL, et al. Two-year follow-up of a primary care-based intervention to prevent and manage childhood obesity: The High Five for Kids study. Pediatr Obes 2017;12:e24-e27.

228. Wright DR, Kenney EL, Giles CM, et al. Modeling the cost effectiveness of child care policy changes in the U.S. Am J Prev Med 2015;49:135-147.

229. Woo Baidal JA, Price SN, Gonzalez-Suarez E, et al. Parental perceptions of a motivational interviewing-based pediatric obesity prevention intervention. Clin Pediatr 2013;52:540-548.

230. Fiechtner L, Block J, Duncan DT, et al. Proximity to supermarkets associated with higher body mass index among overweight and obese preschool-age children. Prev Med 2013;56:218-221.

231. Hohman KH, Price SN, Sonneville K, et al. Can the Internet be used to reach parents for family-based childhood obesity interventions? Clin Pediatr 2012;51:314-320.

232. Gillman M. Improving primary care to prevent childhood obesity. 2006. Available at https://clinicaltrials.gov/ct2/show/NCT00377767 Last accessed October 15, 2019. 
233. Wright DR, Taveras EM, Gillman MW, et al. The cost of a primary care-based childhood obesity prevention intervention. BMC Health Serv Res 2014;14:44.

234. Tomayko EJ, Prince RJ, Cronin KA, Adams AK. The Healthy Children, Strong Families intervention promotes improvements in nutrition, activity and body weight in American Indian families with young children. Public Health Nutr 2016; 19:2850-2859.

235. Tomayko EJ, Prince RJ, Cronin KA, Adams AK. The Healthy Children, Strong Families intervention promotes improvements in nutrition, activity, and body weight in American Indian families with young children-ERRATUM. Public Health Nutr 2017;20:380.

236. LaRowe TL, Adams AK, Jobe JB, et al. Dietary intakes and physical activity among preschool-aged children living in rural American Indian communities before a family-based healthy lifestyle intervention. J Am Diet Assoc 2010;110:1084-1088.

237. Adams AK, LaRowe TL, Cronin KA, et al. The Healthy Children, Strong Families intervention: Design and community participation. J Prim Prev 2012;33:175-185.

238. LaRowe TL, Wubben DP, Cronin KA, et al. Development of a culturally appropriate, home-based nutrition and physical activity curriculum for Wisconsin American Indian families. Prev Chronic Dis 2007;4:A109.

239. Adams AK, Cronin KA. Healthy children, strong families: Obesity prevention for preschool American Indian children and their families. In: Brennan VM, Kumanyika SK, Zambrana RE (eds), Obesity Interventions in Underserved Communities: Evidence and Directions. Johns Hopkins University Press: Baltimore, MD, 2014, pp. 344-352.

240. Adams AK, LaRowe T, Cronin KA, et al. Healthy children, strong families: Results of a randomized trial of obesity prevention for preschool American Indian children and their families. Obesity 2011;19:S110.

241. Tomayko EJ, Prince RJ, Cronin KA, et al. The Healthy Children, Strong Families 2 (HCSF2) randomized controlled trial improved healthy behaviors in American Indian families with young children. Curr Dev Nutr 2019;3(Suppl 2):53-62.

242. Adams AK, Tomayko EJ, Cronin KA, et al. Predictors of overweight and obesity in American Indian families with young children. J Nutr Educ Behav 2019;51:190-198.

243. Berns RM, Tomayko EJ, Cronin KA, et al. Development of a culturally informed child safety curriculum for American Indian families. J Prim Prev 2017;38:195-205.

244. Grant VM, Tomayko EJ, Prince RJ, et al. Understanding correlates of physical activity in american indian families: The healthy children strong families-2 study. J Phys Act Health 2018;15:866-873.

245. Ingram DG, Irish LA, Tomayko EJ, et al. Overnight sleep duration and obesity in $2-5$ year-old American Indian children. $\mathrm{Pe}$ diatr Obes 2018;13:406-412.

246. Tomayko EJ, Prince RJ, Cronin KA, et al. Healthy Children, Strong Families 2: A randomized controlled trial of a healthy lifestyle intervention for American Indian families designed using community-based approaches. Clin Trials 2017;14:152-161.

247. Adams AK. Healthy children, strong families: American Indian Communities Preventing Obesity (HCSF2). 2013. Available at https:/clinicaltrials.gov/ct2/show/record/NCT01776255?term=NCT 01776255\&rank=1 Last accessed October 15, 2019.

248. Tucker JM, DeFrang R, Orth J, et al. Evaluation of a primary care weight management program in children aged $2-5$ years: Changes in feeding practices, health behaviors, and body mass index. Nutrients 2019;11:498.
249. Tucker JM. Evaluation of a primary care weight management program in children aged 2 to 5 years. 2014. Available at https:// clinicaltrials.gov/ct2/show/NCT03487614 Last accessed September 17, 2019.

250. Winter SM, Sass DA. Healthy \& Ready to Learn: Examining the efficacy of an early approach to obesity prevention and school readiness. J Res Child Educ 2011;25:304-325.

251. Blaine RE, Fisher JO, Taveras EM, et al. Reasons low-income parents offer snacks to children: How feeding rationale influences snack frequency and adherence to dietary recommendations. Nutrients 2015;7:5982-5999.

252. Blaine RE, Franckle R, Ganter C, et al. Implementing a childhood obesity intervention in low-income school districts: A mixed methods analysis from the Massachusetts childhood obesity research demonstration (MA-CORD) project. Obes Rev 2016; 17(Suppl 2):152.

253. Davison KK, Falbe J, Taveras EM, et al. Evaluation overview for the Massachusetts Childhood Obesity Research Demonstration (MA-CORD) project. Child Obes 2015;11:23-36.

254. Ganter C, Aftosmes-Tobio A, Chuang E, et al. Stakeholders' perspectives on the effect of a multilevel, multisector intervention on parent engagement in and awareness of childhood obesity prevention and control. FASEB $J$ 2015;29. [Epub ahead of print: DOI: 10.1096/fasebj.29.1_supplement.264.2.]

255. Nelson CC, Colchamiro R, Perkins M, et al. Racial/ethnic differences in the effectiveness of a multisector childhood obesity prevention intervention. Am J Public Health 2018;108: $1200-1206$

256. Taveras EM. Mass in motion kids in the clinical setting. 2014 Available at https://clinicaltrials.gov/ct2/show/record/NCT021106 15?term=NCT02110615\&rank=1 Last accessed October 15, 2019.

257. Taveras EM, Blaine RE, Davison KK, et al. Design of the Massachusetts Childhood Obesity Research Demonstration (MACORD) study. Child Obes 2015;11:11-22.

258. Taveras EM, Perkins M, Anand S, et al. Clinical effectiveness of the massachusetts childhood obesity research demonstration initiative among low-income children. Obesity 2017;25:11591166.

259. Yeh Y, Hartlieb KB, Danford C, Jen KC. Correction to: Effectiveness of nutrition intervention in a selected group of overweight and obese African-American preschoolers. J Racial Ethn Health Disparities 2018;5:562.

260. Sosa ET, Parra-Medina D, He M, et al. ¡Miranos! (Look at Us! We Are Healthy!): Home-based and parent peer-led childhood obesity prevention. Health Promot Pract 2016;17:675-681.

261. Yin Z, Ramirez A, Cordova A, et al. MIRANOS!: A preschool obesity prevention program. Ann Behav Med 2012;43:S191.

Address correspondence to: Lori A.J. Scott-Sheldon, PhD Centers for Behavioral and Preventive Medicine The Miriam Hospital CORO West, Suite 309, 164 Summit Avenue Providence, RI 02906 USA

E-mail: lori_scott-sheldon@brown.edu 